\title{
Improved Eddy-Viscosity Modelling of Turbulent Flow around Porous-Fluid Interface Regions
}

DOI:

10.1007/s11242-019-01357-0

\section{Document Version}

Accepted author manuscript

Link to publication record in Manchester Research Explorer

\section{Citation for published version (APA):}

lacovides, H., Craft, T., \& Al-Aabidy, Q. (2019). Improved Eddy-Viscosity Modelling of Turbulent Flow around Porous-Fluid Interface Regions. Transport in Porous Media. https://doi.org/10.1007/s11242-019-01357-0

\section{Published in:}

Transport in Porous Media

\section{Citing this paper}

Please note that where the full-text provided on Manchester Research Explorer is the Author Accepted Manuscript or Proof version this may differ from the final Published version. If citing, it is advised that you check and use the publisher's definitive version.

\section{General rights}

Copyright and moral rights for the publications made accessible in the Research Explorer are retained by the authors and/or other copyright owners and it is a condition of accessing publications that users recognise and abide by the legal requirements associated with these rights.

\section{Takedown policy}

If you believe that this document breaches copyright please refer to the University of Manchester's Takedown Procedures [http://man.ac.uk/04Y6Bo] or contact uml.scholarlycommunications@manchester.ac.uk providing relevant details, so we can investigate your claim.

\section{OPEN ACCESS}




\title{
Improved Eddy-Viscosity Modelling of Turbulent Flow around Porous-Fluid Interface Regions
}

\author{
Qahtan Al-Aabidy · Timothy J. Craft · Hector Iacovides
}

\begin{abstract}
The RANS modelling of turbulence across fluid-porous interface regions within ribbed channels has been investigated by applying double (both volume and Reynolds) averaging to the Navier-Stokes equations. In this study turbulence is represented by using the Launder and Sharma (1974) low-Reynolds number $k-\varepsilon$ turbulence model, modified via proposals by either Nakayama and Kuwahara (2008) or Pedras and de Lemos (2000), for extra source terms in turbulent transport equations to account for the porous structure. One important region of the flow, for modelling purposes, is the interface region between the porous medium and clear fluid regions. Here, corrections have been proposed to the above porous $\mathrm{drag} /$ source terms in the $k$ and $\varepsilon$ transport equations that are designed to account for the effective increase in porosity across a thin near-interface region of the porous medium, and which bring about significant improvements in predictive accuracy. These terms are based on proposals put forward by Kuwata and Suga (2013), for second-moment closures. Two types of porous channel flows have been considered. The first case is a fully developed turbulent porous channel flow, where the results are compared with DNS predictions obtained by Breugem et al. (2006) and experimental data produced by Suga et al. (2010). The second case is a turbulent solid/porous rib channel flow to examine the behaviour of flow through and around the solid/porous rib, which is validated against experimental work carried out by Suga et al. (2013). Cases are simulated covering a range of porous properties, such as permeability and porosity. Through the comparisons with the available data, it is demonstrated that the extended model proposed here shows generally satisfactory accuracy, except for some predictive weaknesses in regions of either impingement or adverse pressure gradients, associated with the underlying eddy-viscosity turbulence model formulation.
\end{abstract}

Keywords turbulence in porous media $\cdot$ interface porous-fluid region $\cdot$ turbulent flow around a porous rib

\author{
Q. Al-Aabidy \\ E-mail: qahtan.alaabidy@uokufa.edu.iq \\ Present address: University of Kufa \\ T. Craft \\ E-mail: tim.craft@manchester.ac.uk \\ Present address: University of Manchester \\ H. Iacovides \\ E-mail: h.iacovides@manchester.ac.uk \\ Present address: University of Manchester
}




\section{Nomenclature}

\section{Roman Symbols}

$c_{F} \quad$ Forchheimer coefficient

$c_{\varepsilon 1}, c_{\varepsilon 2}, c_{k} \quad$ Non-dimensional turbulence model constants

$c_{\mu} \quad$ Coefficient in the eddy-viscosity

Da Darcy number, $D a=K / H^{2}$

$d_{p} \quad$ Pore diameter

$f_{U}^{\phi}, f_{k}^{\phi}, f_{\varepsilon}^{\phi} \quad$ Damping functions for source terms of porous media

$G_{\varepsilon} \quad$ Generation rate of $\varepsilon$ due to porous media

$G_{k} \quad$ Generation rate of $k$ due to porous media

H Channel height

h Rib height

K Permeability of porous media

$k \quad$ Turbulent kinetic energy

$P_{k} \quad$ Production term

$\mathrm{P} \quad$ Pressure

$R e_{b} \quad$ Bulk Reynolds number, $R e_{b}=U_{b} H /(2 \nu)$

$R_{t} \quad$ Turbulent Reynolds number

$U_{D} \quad$ Darcy or superficial velocity

$y^{\prime} \quad$ Normal distance from the nearest porous surface

$\Delta V \quad$ Total volume

$\Delta V_{f} \quad$ Fluid volume

\section{Greek Symbols}

$\delta_{i j} \quad$ Kronecker delta unit symbol

$\tilde{\varepsilon} \quad$ 'quasi-homogeneous' dissipation rate of the turbulent kinetic energy, $k$

$\nu \quad$ kinematic viscosity

$\nu_{t} \quad$ kinematic turbulent viscosity

$\phi \quad$ Porosity of inhomogeneous porous media $\left(=\Delta V_{f} / \Delta V\right)$

$\phi_{\infty} \quad$ Porosity of homogeneous porous media

$\rho \quad$ Fluid density

\section{Special Characters}
\langle\rangle
Volume average
\langle\rangle$^{f}$
Intrinsic average

\section{Acronyms / Abbreviations}

DNS Direct Numerical Simulation

LES Large Eddy Simulation

LSMNK Launder Sharma Modified by Nakayama and Kuwahara (2008)

LSMPL Launder Sharma Modified by Pedras and de Lemos (2001b)

PPI Pore Per Inch

RANS Reynolds-Averaged Navier-Stokes

REV Representative Elementary Volume

SIMPLE Semi-Implicit Method for Pressure-Linkage Equations

TCL Two-Component-Limit

UMIST Upstream Monotonic Interpolation for Scalar

VAT Volume Averaging Theory 


\section{Introduction}

Porous foams contain highly tortuous flow paths that can significantly intensify the mixing of fluid flow and enhance the heat transfer as a result of extensive surface areas that permit the fluid to be in contact with a large extended area. However, because of the complex flow paths, treating the flow through such media at the pore level requires huge computer power and cost, and even when possible it is limited to simple cases of packed bed type. As a result, the Volume Averaging Theory (VAT) is a common approach for the numerical modelling of flows in porous media (Whitaker 1999).

In many traditional engineering applications the flow in porous media is almost laminar, as a result of the flow resistance caused by the porous structure that leads to relatively low velocities (Antohe and Lage 1997). Turbulence may become appreciable at the pore level, however, if the flow within or around the porous structure is at very high speed, or if the pore scale is larger than the turbulence length scale, i.e. when the pore Reynolds number $R e_{p}$, defined as $R e_{p}=u_{p} d_{p} / \nu$ where $u_{p}$ is the pore velocity scale (intrinsic velocity) and $d_{p}$ is the pore diameter, is sufficiently high (Nakayama and Kuwahara 1999). This is what was visualized by Dybbs and Edwards (1984) who conducted an experimental investigation and found that the flow in the porous media becomes turbulent when the pore Reynolds number was greater than a few hundreds.

Numerical modelling of turbulent flow in porous media is mainly based on a macroscopic approach in which the double-averaging (volume and Reynolds averaging) is used. Macroscopic Navier-Stokes equations can be derived by two different methodologies: either time-averaging the volume-averaged Navier-Stokes equations, or volume-averaging the Reynolds-averaged Navier-Stokes equations. Lee and Howell (1987), Getachew et al. (2000) and (Antohe and Lage 1997) used the first methodology to obtain macroscopic $k-\varepsilon$ transport equations for treating the turbulence in porous media. However, since a highly permeable medium was considered by Lee and Howell (1987), no additional terms were included related to porosity in the turbulence equations. The second methodology, which is based on using the Reynolds averaging first, has been more widely used in dealing with turbulent flows, for example in the studies of Masuoka and Takatsu (1996); Nakayama and Kuwahara (1999); Finnigan (2000); Pedras and de Lemos (2000) and Nikora et al. (2007).

Pedras and de Lemos (2000) proved that the momentum equation is not affected by the order of averaging, although the two approaches do lead to different definitions of the turbulent kinetic energy. Nakayama and Kuwahara (1999) believed that the Reynolds averaging should be performed first in order to detect the turbulence level at the porous scale, since this level of turbulence is unlikely to be detected in the case of starting with volume averaging. The final forms of macroscopic turbulent transport equations obtained by Pedras and de Lemos (2000) and Nakayama and Kuwahara (1999) shared similar terms to those found for the non porous media, but also contained extra production and dissipation rate terms due to the presence of porous structure.

Kuwata and Suga (2013) used a sophisticated turbulence model for treating three unknown elementary second moment terms that appear in the Navier-Stokes equations after applying double (both volume and Reynolds) averaging. These terms are namely the dispersive covariance and the volume averaged Reynolds stress, which is decomposed into the macro-scale and micro-scale(sub-filter scale) Reynolds stress. The TCL(two-component-limit) second moment closure and a one-equation eddy viscosity model were used for modelling macro-scale and micro-scale Reynolds stresses, respectively, while the dispersive covariant was modelled via a form based on the Smagorinsky scheme typically used to represent sub-grid-scale stresses in Large Eddy Simulation (LES). In addition to this model they proposed an economical multi-scale $k-\varepsilon$ model for industrial applications. Mößner and Radespiel (2015) used Reynolds averaging of volume-averaged Navier-Stokes equations, justifying its use in their study by showing good agreement with DNS data from Breugem et al. (2006).

Roughened surfaces, such as those formed by placing ribs on the surface, across the flow direction, effectively enhance the heat transfer due to flow separation and reattachment behind each rib that disrupts 
the thermal boundary layer. In the case of a surface roughened by a solid rib, however, a hot spot occurs at the circulation zone, leading to deterioration of heat transfer around the rib region (Iacovides and Raisee 1999 Iacovides and Launder 2007). To overcome this issue, several investigations have been performed using different techniques to change the geometry of the rib, and the use of perforated ribs is one of these techniques. Wang et al. (2010) conducted experimental PIV measurements to investigate the turbulent flow in a channel with solid or perforated ribs. It was observed that the recirculation bubble behind the perforated rib was smaller compared to the solid rib. The Reynolds shear stress behind the perforated rib was also reduced significantly compared to that behind a solid rib, and that in turn reduced the pressure losses. Similar findings were numerically obtained by Liou et al. (2002) and experimentally by Hwang (1998). The former investigated turbulent flow and heat transfer in a channel with periodic slit ribs by using a highReynolds-number Reynolds stress model. The rib open-area ratio ranged between $0 \%$ to $44 \%$. The results showed a decrease of the recirculation zone size behind the rib when the open-area ratio was increased from $0 \%$ to $44 \%$. It was found that with the large rib open-area ratio, $40 \%$, minimum friction loss, minimum pressure drop, and best heat transfer augmentation under the same pumping power condition was achieved. Hwang (1998) used hot-wire anemometry to measure the distributions of velocity and turbulence intensity of turbulent flow in a rectangular duct with slit and solid ribs mounted on one side wall. Panigrahi et al. (2006) and Panigrahi et al. (2008) used the PIV technique for streamwise and crossstream measurements to understand the turbulent flow structure around ribs with different inclination slits. The results also showed a reduction in the reattachment length behind the slit ribs compared to the solid ribs. Nuntadusit et al. (2012) investigated the effect of perforation and hole inclination in ribs on the flow and heat transfer characteristics. The heat transfer results were obtained experimentally via the thermostat-chromic liquid crystal technique, while the velocity distributions were predicted numerically by using the commercial code ANSYS FLUENT coupled with a high-Re $k-\varepsilon$ model and wall functions. The results showed that the minimum recirculation zone and best heat transfer augmentation was obtained with the largest inclination angle.

Leu et al. (2008) performed an experimental investigation of turbulent flow in a channel with a rib mounted at the bottom by using the Acoustic Doppler velocimeter (ADV). Ribs of three different structures were examined, whose geometric porosities were $0 \%, 34.9 \%$, and $47.5 \%$. The resulting measurements indicated that increasing porosity led to greater flow penetration through the porous rib and decreased the flow over the rib. It was also observed that the recirculation region was decreased in size and shifted downstream of the rib when the porosity was increased. The maximum streamwise turbulent intensity was decreased by $37 \%$ in case of higher porosity(47.5\%) compared to the solid rib.

Suga et al. (2013) performed PIV measurements for turbulent flow in a channel half-filled with a porous layer with a square rib, of half the clear channel height, mounted on top of this layer. Two kinds of square rib, namely a solid smooth rib and a porous rib, which was made of the same material of that for the porous layer, were tested. Three kinds of foamed ceramics were employed with the same porosity, $80 \%$, but with different permeability based on different numbers of pores per inch (PPI). The values of permeability, normalized by rib height, were $0.89 \times 10^{-4}, 1.47 \times 10^{-4}$ and $3.87 \times 10^{-4}$, which were for PPI=20, 13 and 6 , respectively. Consideration was given to the effects of permeability of the rib and the layer on the separation and reattachment of the flow at bulk Reynolds numbers ranging from $10^{3}$ to $10^{4}$. The measurements indicated that the turbulence levels and Reynolds shear stress in the wake region behind the rib were much higher than those in fully developed porous channel flows. In the solid rib flows, the turbulent energy levels weakened behind the rib and the recirculation bubble reduced and shifted downstream and eventually vanished as the permeability or the Reynolds number were increased. The turbulence levels, however, were lower than those of turbulent flow over a solid rib mounted on a solid wall. In the porous rib flows, the turbulent energy levels and shear stress were lower, due to bleeding of flow through the porous rib. A recirculation bubble hardly existed in the clear channel behind the rib in the highest permeability case. A reversed flow region did exist in the porous layer behind the solid rib, even in the high permeability case, while it did not appear in the case of the porous rib. 
Chan et al. (2010) numerically studied turbulent flow over a porous rib by using the Pedras and de Lemos (2001b) model. Their predictions were in a good agreement with the experimental study of Leu et al. (2008). Kuwata et al. (2014) used their multi-scale $k-\varepsilon$ model to investigate turbulent flow around a porous rib mounted on a porous layer that half filled a channel. The results showed that the predictions were satisfactory and in good agreement with the experimental study of Suga et al. (2013). Further downstream of the rib, however, streamwise velocity and turbulent energy predictions were not satisfactory. That was attributed to the weakness of the eddy viscosity models behind obstacles and in redeveloping flows.

Another application of flow through porous media has been the study of turbulent flows within and above vegetation regions, with both experimental and numerical approaches. The vegetation regions have been considered as porous regions. Dunn et al. (1996) measured flow and turbulence structure through vegetative canopy channels by employing an acoustic Doppler velocimetry. The vegetation regions were simulated by rigid and flexible cylinders. Nezu and Sanjou (2008) used laser Doppler anemometry (LDA) and particleimage velocimetry (PIV) measurements, in addition to LES calculations, to investigate turbulence structures and coherent motions in vegetative canopy channels. The vegetation regions were modelled by using rigid strip plates.

In any of the applications mentioned above, at the fluid/porous interface region, there is a complex interaction between the fluid in the porous region with that in the non-porous region. This means that no-slip boundary conditions are no longer valid at the fluid-porous interface. Beavers and Joseph (1967) found an empirical relationship to relate the slip velocity to the exterior flow. Ochoa-Tapia and Whitaker (1995) improved the stress jump condition suggested by Beavers and Joseph (1967) and proposed another stress jump condition for the boundary between porous media and a homogeneous fluid to link the Darcy's law including Brinkman term with Stokes' equations to obtain a continuous volume-averaged flow field. Silva and de Lemos (2003a) employed this stress jump at the interface for laminar flow cases. Continuity in the diffusion of turbulent energy boundary conditions was utilised for turbulent flow cases in another study (Silva and de Lemos 2003b). Chan et al. (2007) used Volume averaging of Reynolds averaged Navier-Stokes equations and the Pedras and de Lemos (2001b) model to study turbulent flow over a porous medium. Continuity boundary conditions were applied for velocity, pressure and shear stress at the fluid/porous interface. The numerical calculations were compared with the experiments reported by Prinos et al. (2003). The results showed that penetration of turbulence through the porous region is directly related to the permeability and porosity of the porous region.

A diffusion-jump model for turbulent energy through the fluid/porous interface was proposed and discussed by De Lemos (2005) and De Lemos (2009), where negative and positive jump coefficients were examined. Chandesris and Jamet (2006) derived a stress-jump boundary condition at the fluid/porous interface for laminar flow regime. It was found that the stress jump coefficient was dependent on the internal structure of the transition zone. This boundary condition was used later, Chandesris and Jamet (2009), by utilising a two-step up-scaling framework to impose boundary conditions at the fluid/porous interface for a turbulent flow regime. Pokrajac and Manes (2008) also derived a stress-jump condition for turbulent flow over a rough fluid/porous interface. Details on various approaches to treat the flow at the fluid/porous interface were also discussed. Kuznetsov (2004) investigated the effect of the roughness of the fluid/porous interface on the turbulent forced convection through it. This was conducted by extending the approach proposed in previous studies, such as Kuznetsov and Xiong (2003), which assumed a hydraulically smooth fluid/porous interface. A $k-\varepsilon$ model was utilised in the turbulent flow region over the porous region, while in the flow near the interface a $k-l$ model was used. The results showed that, in the case of $D a=10^{-2}$, the velocity profiles for the rough interface differed from those for the smooth interface. In the case of $D a=10^{-4}$, on the other hand, the velocity profiles were identical for both rough and smooth interfaces. More recently, Kuwata and Suga (2013) proposed a different approach within the context of a second-moment closure. This approach was to introduce damping functions to relax the source terms linked to the porous structure in the governing equations as the fluid-porous interface region is approached. 
In the open literature, the majority of the researches have used $k-\varepsilon$ Eddy Viscosity Models for turbulent flows in porous media. Although, more recently, Suga and his co-workers, e.g. (Kuwata and Suga 2013), have used quite sophisticated stress transport models, the majority of research reported has employed simpler eddy-viscosity models. Due to their widespread use in industry, the present study has also explored modelling techniques within the eddy-viscosity framework, whilst recognising some of the inherent limitations in applying such schemes to certain flow regions.

In this study, the double-averaging technique has been used to model the flow through porous media, with surrounding clear fluid. The Launder and Sharma low-Reynolds-number $k-\varepsilon$ turbulence model has been used, with modifications proposed by Nakayama and Kuwahara (2008) and Pedras and de Lemos (2000, 2001b) to mimic the flow inside the porous media. To improve predictions close to the fluid/porous interface, additional damping terms are introduced to the porous source terms in this region, to relax the resistance of the porous medium from the homogeneous porous region to the clear fluid zone across the distance of the mean pore diameter. These damping functions are modified forms of those proposed by Kuwata and Suga (2013). Results from the use of the current extended turbulence models are compared with DNS data by Breugem et al. (2006) and experimental data by Suga et al. $(2010,2013)$ to validate the models.

\section{Macroscopic Mathematical Formulation}

\subsection{Macroscopic Navier-Stokes Equations}

The turbulent flow over porous walls or around solid/porous ribs is described by the conventional RANS equations, while the flow through porous regions is described by the Brinkman-Forchheimer-extended Darcy model. This latter formulation accounts for viscous effects (Darcy term), form drag (Forchheimer term) and the Brinkman term (viscous diffusion) (Vafai and Tien 1981). The continuity and mean momentum equations can be written as (Pedras and de Lemos $2001 \mathrm{~b})$ :

$$
\begin{gathered}
\frac{\partial\left\langle U_{i}\right\rangle}{\partial x_{i}}=0 \\
\frac{\partial\left\langle U_{i}\right\rangle}{\partial t}+\frac{\partial}{\partial x_{j}}\left(\frac{\left\langle U_{j}\right\rangle\left\langle U_{i}\right\rangle}{\phi}\right)=-\frac{1}{\rho} \frac{\partial \phi\langle P\rangle^{f}}{\partial x_{i}}+\frac{\partial}{\partial x_{j}}\left[\nu\left(\frac{\partial\left\langle U_{i}\right\rangle}{\partial x_{j}}+\frac{\partial\left\langle U_{j}\right\rangle}{\partial x_{i}}\right)-\phi \overline{\left\langle u_{i} u_{j}\right\rangle^{f}}\right] \\
-f_{U}^{\phi}\left[\frac{\nu \phi}{K}\left\langle U_{i}\right\rangle+\frac{c_{F} \phi}{\sqrt{K}} \sqrt{\left\langle U_{k}\right\rangle\left\langle U_{k}\right\rangle}\left\langle U_{i}\right\rangle\right]
\end{gathered}
$$

where $K$ is the permeability of the porous medium, which is defined as a measure of its ability to permit fluid flow through it. It represents a measure of the interconnected pores inside porous medium that allows fluid to penetrate within these pores. This means it is a property of hydraulic conductance of the flow through the porous medium, and identifies the surface area that is open to the flow $(\overline{K a v i a n y} 1991) . c_{F}$ is the Forchheimer coefficient, or the form drag coefficient, that depends on the nature of the matrix structure of the porous media. The permeability, $K$, and the Forchheimer coefficient, $c_{F}$, can be determined using the Darcy-Forchheimer equation via measurement of the total pressure drop per unit length of the porous medium (Straatman et al. 2007, Naaktgeboren et al. 2012). $\phi\langle P\rangle^{f}$ is the superficial average pressure of the fluid, and $\phi$ is the porosity of the porous medium, which is defined as the pores' volume fraction. In other words, the porosity can be defined as the ratio of the volume of voids space (occupied by fluid) to the total volume of the solid matrix, and can be mathematically defined as:

$$
\phi=\frac{\Delta V_{f}}{\Delta V}
$$


The above mean that the characteristics of a porous medium are fully defined by the values of $K, c_{F}$ and $\phi$. The relationship between the superficial $\langle\Psi\rangle$ and intrinsic $\langle\Psi\rangle^{f}$ property is $\langle\Psi\rangle=\phi\langle\Psi\rangle^{f}$. The superficial averaged value (or the total volume averaged value) of a variable $\Psi$, is defined as follows (Kaviany 1991):

$$
\langle\Psi\rangle=\frac{1}{\Delta V} \int_{\Delta V_{f}} \Psi d v
$$

while $\langle\Psi\rangle^{f}$ represents the intrinsic averaged value (or fluid volume averaged value) of a variable $\Psi$, defined as follows:

$$
\langle\Psi\rangle^{f}=\frac{1}{\Delta V_{f}} \int_{\Delta V_{f}} \Psi d v
$$

Similarly:

$$
\left\langle U_{i}\right\rangle=\phi\left\langle U_{i}\right\rangle^{f}
$$

where $\left\langle U_{i}\right\rangle$ is the total volume averaged velocity (or Darcy velocity) which is superficial velocity.

The term $f_{U}^{\phi}$ in equation (2) is introduced below, in Section 2.3 to account for the near-interface effects in the porous media. When the porosity and permeability are extremely high (and the porous matrix effectively disappears), the generalized model equation (2) reverts to the traditions RANS equations.

The macroscopic Reynolds stress tensor, $\phi \overline{\left\langle u_{i} u_{j}\right\rangle^{f}}$, appearing in equation (2) has been modelled in analogy with the Boussinesq concept for clear fluid (non-porous), which is a linear stress-strain relation, as follows (Pedras and de Lemos 2001b):

$$
\phi \overline{\left\langle u_{i} u_{j}\right\rangle^{f}}=-\nu_{t \phi}\left(\frac{\partial\left\langle U_{i}\right\rangle}{\partial x_{j}}+\frac{\partial\left\langle U_{j}\right\rangle}{\partial x_{i}}\right)+\frac{2}{3} \phi\langle k\rangle^{f} \delta_{i j}
$$

where $\langle k\rangle^{f}$ is the intrinsic turbulent kinetic energy, and $\nu_{t \phi}$ is the macroscopic turbulent viscosity, modelled similarly to that in the clear fluid as:

$$
\nu_{t \phi}=f_{\mu} c_{\mu} \frac{\langle k\rangle^{f^{2}}}{\langle\tilde{\varepsilon}\rangle^{f}}
$$

where $c_{\mu}$ is a constant with the usual value of 0.09 and $f_{\mu}$ a near-wall damping term, here taking the form proposed by Launder and Sharma (1974) of exp $\left[-3.4 /\left(1+R e_{t} / 50\right)^{2}\right]$.

\subsection{Macroscopic equations for $k$ and $\tilde{\varepsilon}$}

Nakayama and Kuwahara (1999) and Pedras and de Lemos (2000) conducted numerical experiments for turbulent flow around periodically arranged square rods and circular rods, respectively. The final forms they adopted for the macroscopic turbulent kinetic energy and its dissipation rate equations, after applying the volume-averaging operator for microscopic $k-\varepsilon$ equations inside a Representative Elementary Volume $\mathrm{REV}$, can be written as follows:

$$
\begin{aligned}
\frac{\partial \phi\langle k\rangle^{f}}{\partial t}+\frac{\partial\left(\left\langle U_{j}\right\rangle\langle k\rangle^{f}\right)}{\partial x_{j}}= & \frac{\partial}{\partial x_{j}}\left[\left(\nu+\frac{\nu_{t \phi}}{\sigma_{k}}\right) \frac{\partial \phi\langle k\rangle^{f}}{\partial x_{j}}\right]+P_{k}^{f}+f_{k}^{\phi} G_{k} \\
& -\left[\phi\langle\tilde{\varepsilon}\rangle^{f}+2 \nu\left(\frac{\partial\left(\phi\langle k\rangle^{f}\right)^{\frac{1}{2}}}{\partial x_{j}}\right)^{2}\right]
\end{aligned}
$$




$$
\begin{aligned}
\frac{\partial \phi\langle\tilde{\varepsilon}\rangle^{f}}{\partial t}+\frac{\partial\left(\left\langle U_{j}\right\rangle\langle\tilde{\varepsilon}\rangle^{f}\right)}{\partial x_{j}}= & \frac{\partial}{\partial x_{j}}\left[\left(\nu+\frac{\nu_{t \phi}}{\sigma_{\varepsilon}}\right) \frac{\partial \phi\langle\tilde{\varepsilon}\rangle^{f}}{\partial x_{j}}\right]+c_{\varepsilon 1} \frac{\langle\tilde{\varepsilon}\rangle^{f}}{\langle k\rangle^{f}} P_{k}^{f} \\
& +c_{\varepsilon 2}\left(f_{\varepsilon}^{\phi} G_{\varepsilon}-f_{1} \frac{\langle\tilde{\varepsilon}\rangle^{f}}{\langle k\rangle^{f}} \phi\langle\tilde{\varepsilon}\rangle^{f}\right)+2 \nu \nu_{t}\left(\frac{\partial^{2}\left\langle U_{i}\right\rangle}{\partial x_{j} \partial x_{k}}\right)^{2}
\end{aligned}
$$

where $P_{k}^{f}=-\phi \overline{\left\langle u_{i} u_{j}\right\rangle^{f}} \frac{\partial\left\langle U_{i}\right\rangle^{f}}{\partial x_{j}}$ is defined as the production rate of $\langle k\rangle^{f}, f_{1}$ is a viscous damping term for the low-Reynolds-number $k-\varepsilon$ Launder and Sharma (1974) model, taken as $f_{1}=1.0-0.3 \exp \left(-R_{t}{ }^{2}\right)$. $G_{k}$ and $G_{\varepsilon}$ represent respectively the extra generation rate of $\langle k\rangle^{f}$ and the extra production rate of $\langle\tilde{\varepsilon}\rangle^{f}$ due to the presence of the porous structure. These extra source terms were modelled in different forms in the two studies, as shown in the Table 1, where $c_{k}$ is constant equal to 0.28. Pedras and de Lemos (2001a) included the source terms in the $k$ and $\varepsilon$ equations based on expressions proportional to the mean macroscopic velocity and to $\langle k\rangle^{f}$ (or $\langle\tilde{\varepsilon}\rangle^{f}$ ) itself. These source terms were proposed according to the gradients of local macroscopic velocity within the pore that contribute to increasing turbulence levels as the flow resistance increases (by increasing $\phi / K)$. Nakayama and Kuwahara (2008) proposed the extra source terms for the production and dissipation rate, due to presence of porous structure, based on the mean flow kinetic energy balance within the pore. The functions $f_{k}^{\phi}$ and $f_{\varepsilon}^{\phi}$ are the near-interface damping terms introduced in the present study, and are described in Section 2.3 below.

\section{Table 1:}

Expressions for extra production terms for turbulent kinetic energy and its dissipation rate

\begin{tabular}{ccc}
\hline & $G_{k}$ & $G_{\varepsilon}$ \\
\hline Pedras and de Lemos $2001 \mathrm{~b})$ & $c_{k} \frac{\phi\langle k\rangle^{f}}{\sqrt{K}} \sqrt{\left\langle U_{k}\right\rangle\left\langle U_{k}\right\rangle}$ & $c_{k} \frac{\phi\langle\tilde{\varepsilon}\rangle^{f}}{\sqrt{K}} \sqrt{\left\langle U_{k}\right\rangle\left\langle U_{k}\right\rangle}$ \\
\hline Nakayama and Kuwahara (2008) & $\frac{c_{F}}{\sqrt{K}}\left(\left\langle U_{k}\right\rangle\left\langle U_{k}\right\rangle\right)^{3 / 2}$ & $\frac{c_{F}}{K} \sqrt{\frac{c_{\mu}}{2 \phi}}\left(\left\langle U_{k}\right\rangle\left\langle U_{k}\right\rangle\right)^{2}$ \\
\hline
\end{tabular}

\subsection{Modification for Porous-Fluid Interface Regions}

The equations introduced in the previous section are valid for the porous region, whilst in the clear fluid region the additional terms in the momentum and turbulence terms clearly vanish. However, the additional terms are based on spatially uniform porous medium properties, whilst there will be a thin layer at its interface with the clear fluid where the effective porosity will increase as the clear fluid region is approached, as a result of the pore structure typically becoming more "open" very close to the boundary of the porous medium. As will be seen below, if this feature is not accounted for then the above models tend to significantly overpredict the levels of turbulence around the fluid/porous interface. Kuwata and Suga (2013) accounted for this effect in their stress transport model by introducing functions to increase the effective porosity and damp the porosity-related source terms in the macroscopic RANS equations across a thin layer next to the interface. A similar approach has been taken here, with damping functions optimized for use with the above set of models. The porosity is therefore taken as:

$$
\phi=\phi_{\infty}+\left(1-\phi_{\infty}\right) \exp \left(-N_{\phi} y^{\prime} / d_{p}\right)
$$

where $\phi_{\infty}$ is the porosity of the homogeneous porous medium and $y^{\prime}$ is the normal distance from the nearest porous surface. The coefficient $N_{\phi}$, which is taken as 4 , is chosen so that the porosity varies only within the 
distance of the mean pore diameter, $d_{p}$, from the interface. The drag terms, in the momentum equation (2), and other additional terms in the turbulence transport equations $(9) \&(10)$, are respectively multiplied by the following functions:

$$
\begin{aligned}
& f_{U}^{\phi}=1-\exp \left(-1.03\left(y^{\prime} / \max (0.004, \sqrt{K})\right)^{1 / 2}\right) \\
& f_{k}^{\phi}=1-\exp \left(-1.21\left(y^{\prime} / \max (0.004, \sqrt{K})\right)^{5 / 2}\right) \\
& f_{\varepsilon}^{\phi}=1-\exp \left(-\left(1.0+R_{t} / 58.51\right)\left(y^{\prime} / \max (0.004, \sqrt{K})\right)^{2}\right)
\end{aligned}
$$

where $R_{t}\left(=\left(\phi\langle k\rangle^{f}\right)^{2} /\left(\nu \phi\langle\tilde{\varepsilon}\rangle^{f}\right)\right)$ is the turbulent Reynolds number. The damping function of $\tilde{\varepsilon}$, $f_{\varepsilon}^{\phi}$, is taken to vary also with turbulence Reynolds number, across a thin layer next to the interface, to fit the available data. The above functions have been optimised for use within the present $k-\varepsilon$ framework over a range of different cases with different porous medium properties and Reynolds numbers.

\section{Results and Discussions}

The calculations presented in this study have been carried out by using an in-house finite volume code STREAM developed in Manchester by Lien and Leschziner (1994a). It employs a non-orthogonal and body-fitted grid system in which all transported properties are stored in a fully collocated manner. The SIMPLE pressure-correction algorithm of Patankar and Spalding (1972) is used to evaluate the pressure field in addition to Rhie and Chow (1983) interpolation to avoid pressure oscillations. Advective volumeface fluxes are approximated using the second order-accurate UMIST scheme, Lien and Leschziner (1994b). The computations are run until the residuals fall to $\mathcal{O}\left(10^{-6}\right)$. Results are presented from the use of the current extended turbulence models, referred to as LSMNK in case of that based on the Nakayama and Kuwahara (2008) turbulence model and LSMPL in case of that based on the Pedras and de Lemos (2000, 2001b) model.

\subsection{Turbulent Porous Channel Flows}

The first case considered for the validation of the modified model is that of a fully developed plane channel flow over a porous layer, which was examined using DNS data generated by Breugem et al. (2006) and experimentally by Suga et al. (2010). Such flows are encountered in a wide range of engineering and environmental problems such as metal foam heat exchangers, catalytic converters, flows in oil wells, flows over forests and porous river beds.

\subsubsection{Case description}

Figure 1a shows the channel geometry, with a solid top wall and a permeable lower layer. The total channel height is $\mathrm{H}$, and the bottom impermeable wall is covered by a porous layer of half the channel height. A block-structured grid of around $30(x) \times 300(y)$ cells was used, with grid nodes concentrated towards the fluid/porous interface and solid walls, as shown in Figure $1 \mathrm{~b}$ Tests with more refined grids confirmed the distribution adopted to be sufficient. The computational mesh is refined near to the impermeable boundaries to ensure that the near-wall non-dimensional wall distance $y^{+}$values are less than unity.

The bulk Reynolds number is defined as $R e_{b}=U_{b} H /(2 \nu)$ based on the bulk velocity $U_{b}$ of the flow in the clear channel region and its height $H / 2$, as proposed by Suga et al. (2010). Periodic boundary conditions 


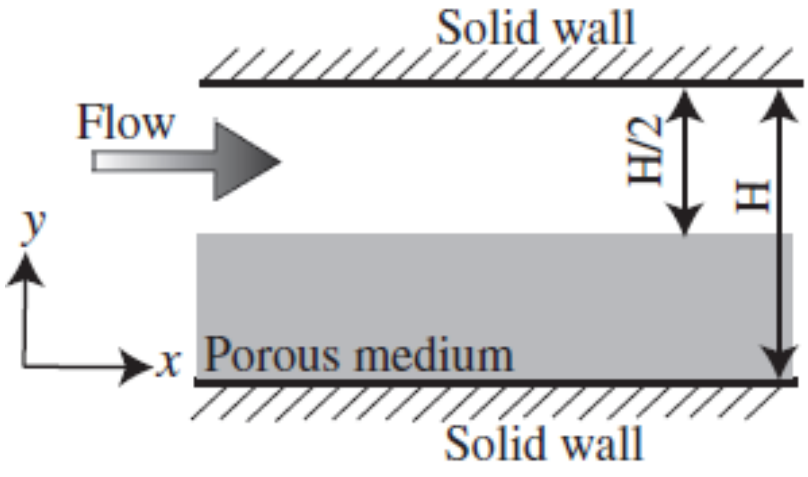

(a) schematic of the computational domain.

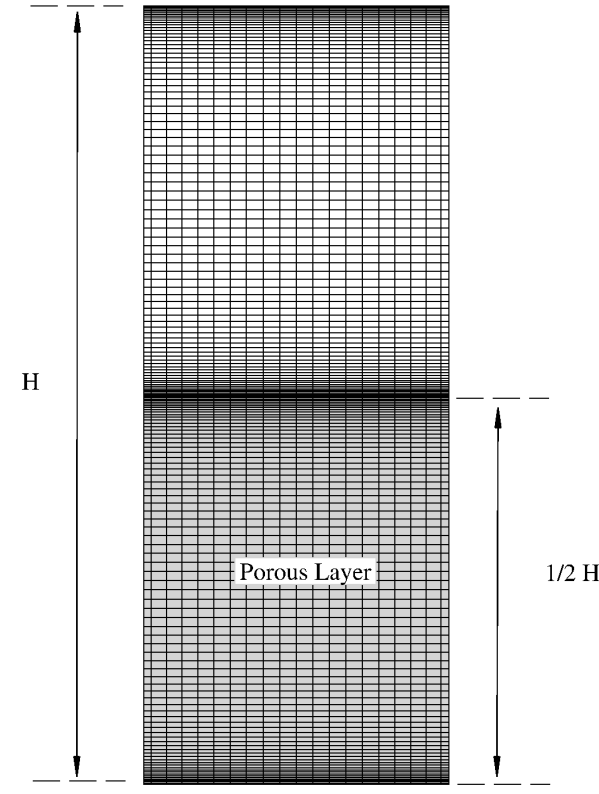

(b) Computational grid.

Fig. 1: Configuration of computational domain and grid for a turbulent porous plane channel flows.

are imposed between the upstream and downstream boundaries with no-slip boundary conditions at the impermeable walls. The imposed streamwise pressure gradient is adjusted in an iterative fashion to result in the desired bulk flow rates.

Simulations have been performed covering a range of porosity, permeability and Reynolds numbers, matching the available DNS and experimental data, as shown in Table 2. For the available DNS data, the values of mean pore diameter $d_{p} / H$ are a surrogated effective value of the inscribed sphere diameter of the cubic particles that were considered in the DNS study (Kuwata and Suga 2013). For simplicity in referring to the considered cases, the porosity is used to distinguish them in cases of the DNS studies, while for the experimental cases PPI and Reynolds number are used.

Table 2: Parameters of the porous media of experimental and DNS studies.

\begin{tabular}{|c|c|c|c|c|c|c|c|}
\hline Case & $R e_{b}$ & $\phi$ & $D a$ & PPI & $c_{F}$ & $d_{p} / H$ & Reference \\
\hline E80 & 5500 & $80 \%$ & $1.78 \times 10^{-6}$ & - & 0.375 & 0.018 & Breugem et al. (2006) \\
\hline E95 & 5500 & $95 \%$ & $4.75 \times 10^{-5}$ & - & 0.295 & 0.011 & Breugem et al. $(\overline{\overline{2006}})$ \\
\hline$\# 20 \mathrm{~L}$ & 2870 & $82 \%$ & $6.20 \times 10^{-6}$ & 20 & 0.17 & 0.030 & Suga et al. (2010) \\
\hline \#20M & 5500 & $82 \%$ & $6.20 \times 10^{-6}$ & 20 & 0.17 & 0.030 & Suga et al. $(2010)$ \\
\hline$\# 20 \mathrm{H}$ & 10200 & $82 \%$ & $6.20 \times 10^{-6}$ & 20 & 0.17 & 0.030 & Suga et al. $(\overline{2010})$ \\
\hline \#13M & 5300 & $81 \%$ & $9.93 \times 10^{-6}$ & 13 & 0.10 & 0.048 & Suga et al. $(\overline{2010})$ \\
\hline$\# 13 \mathrm{H}$ & 10200 & $81 \%$ & $9.93 \times 10^{-6}$ & 13 & 0.10 & 0.048 & Suga et al. $(\overline{2010})$ \\
\hline$\# 6 \mathrm{M}$ & 5400 & $80 \%$ & $2.60 \times 10^{-5}$ & 6 & 0.095 & 0.065 & Suga et al. $(\overline{2010})$ \\
\hline$\# 6 \mathrm{H}$ & 9500 & $80 \%$ & $2.60 \times 10^{-5}$ & 6 & 0.095 & 0.065 & Suga et al. $(\overline{2010})$ \\
\hline
\end{tabular}




\subsubsection{Results}

For validation purposes, profiles are presented across both porous and clear fluid regions of the channel of the mean velocity, normalized by bulk velocity in the clear channel, and turbulent kinetic energy, and Reynolds shear stress, normalized by friction velocity $U_{\tau}$ at the (impermeable) top wall. From the experimental and DNS results, it can be generally seen that penetration of fluid and some turbulence structures across the porous boundary causes less of a wall-blocking effect there (particularly on the wall-normal velocity component) than would be found at a solid wall. As a result, Suga et al. (2010) noted that the flow becomes turbulent at a lower Reynolds number than would be expected in a clear plane channel. The measurements show higher turbulence levels in the clear fluid near the porous interface than near the solid wall, with the peak levels increasing as the porosity increases, together with a highly asymmetric mean velocity across the clear fluid part of the channel. These features can be explained by noting that, since the flow is fully developed, the momentum equation implies that the total shear stress must increase linearly across the clear fluid part of the channel (as it would for a purely clear fluid channel flow), and the results show the turbulent shear stress doing so across the core region. Clearly, the mean velocity and turbulent shear stress must both fall to zero at the solid wall, however they do not do so at the porous interface. Consequently, there is not a rapid growth of viscous shear stress as the interface is approached, and the velocity gradient here is lower than near the solid wall, leading to the asymmetric profile seen in the data. The peak of the velocity profile is displaced towards the top wall, and corresponds to the location at which the Reynolds shear stress is zero in the clear part of the channel.

Considering first the case with the highest porosity and permeability levels (E95) compared to other studied cases, Figure 2 shows profiles of mean velocity, turbulent kinetic energy and turbulent shear stress across the channel. As can be seen, the original Pedras and de Lemos (2001b) and Nakayama and Kuwahara (2008) model forms return too high levels of turbulence around the porous/clear fluid interface, with the Pedras and de Lemos (2001b) form resulting in high $k$ levels right across the porous region, and both leading to rather too much asymmetry in the mean velocity profile across the clear fluid region. The addition of the proposed near-interface terms in the porosity and turbulence model equations brings the turbulence levels down to closely match the DNS data, with the resulting mean velocity also giving a good fit to the DNS. It can be also seen that there is a very good agreement between the current calculations from both models and those obtained by Kuwata et al. (2014) with a multi-scale $k-\varepsilon$ turbulence model, as shown in Figure 2,

In the lower permeability and porosity cases of \#6M and \#6H the proposed models still perform well (although plots are not included, for space reasons). As the permeability is further decreased (cases \#13M, \#13H), Figure 3 shows that both models still perform quite well at the higher Reynolds number of 10,200. However, whilst use of the LSMPL model also results in good agreement with the data at the lower Reynolds number, (case\#13M), the LSMNK variant now returns too high levels of turbulence. A similar behaviour is seen for the even lower permeability cases, \#20M and \#20H, although not shown here, to avoid repetition. This difference in model behaviour becomes even more apparent as the permeability is reduced further (case E80, Figure 4). Although the DNS data still show some asymmetry in the mean velocity profile, it is now much less than in the earlier cases. The LSMPL model captures this behaviour, and still results in good agreement with the data, whilst the LSMNK scheme returns very high turbulence levels, and a correspondingly asymmetric mean velocity profile. The cause of the difference in model behaviour at low Reynolds numbers would appear to lie in the forms adopted for the modelled source terms $G_{k}$ and $G_{\varepsilon}$. Based on arguments of how terms in the turbulent kinetic energy equation should scale, Nakayama and Kuwahara (2008) formulated their additional source terms to depend on the mean flow kinetic energy balance within voids of porous media, whereas the forms proposed by Pedras and de Lemos $(2001 \mathrm{~b})$ are proportional to the macroscopic velocity and the local turbulent kinetic energy and its dissipation rate levels. From the results it would appear that the latter formulation, responding directly to the actual values of $k$ and $\varepsilon$, gives the better predictions over the range of Reynolds numbers and permeability values tested here. 


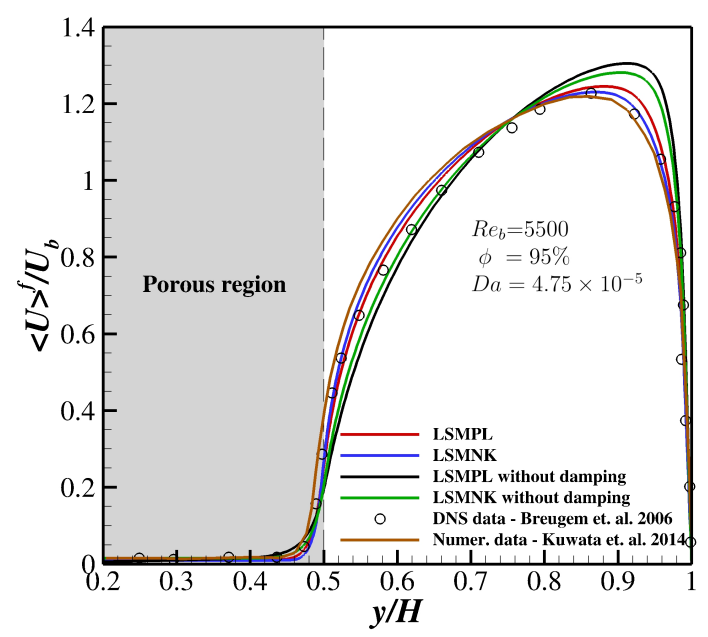

(a) Streamwise mean velocity profile

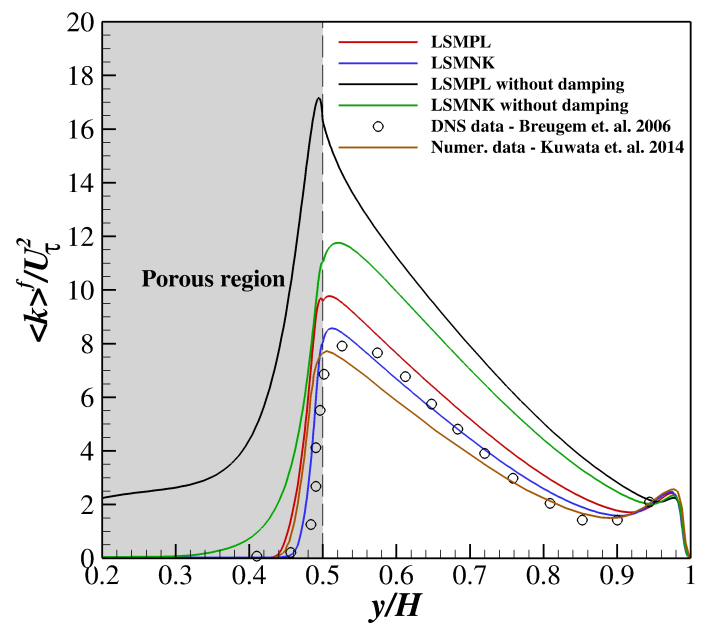

(b) Turbulent kinetic energy profile

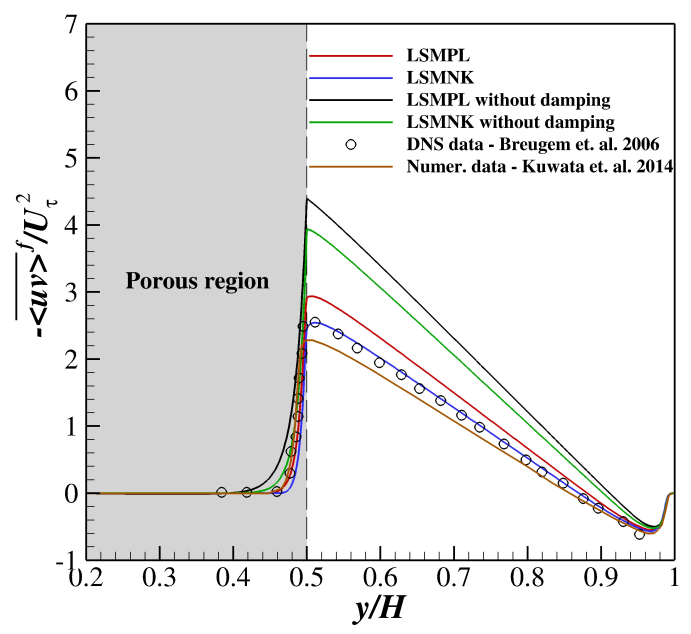

(c) Reynolds shear stress profile

Fig. 2: Comparison of the current predictions with both the DNS data Breugem et al. (2006) and the calculations of Kuwata et al. (2014) for the high porosity plane channel flow , case E95. 


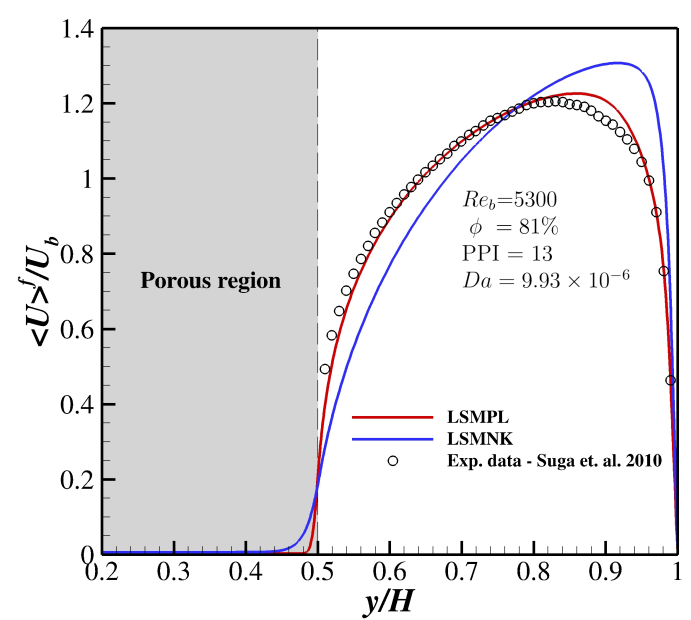

(a) Streamwise mean velocity profile of Case\#13M

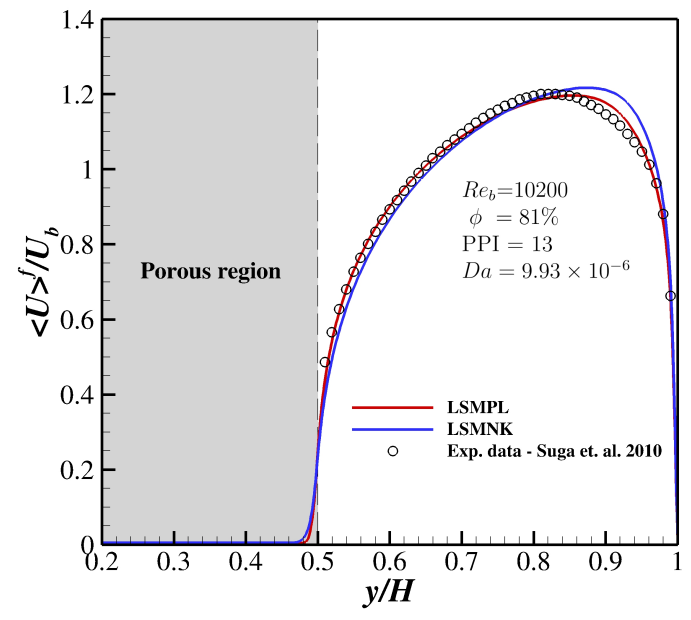

(c) Streamwise mean velocity profile of Case\#13H

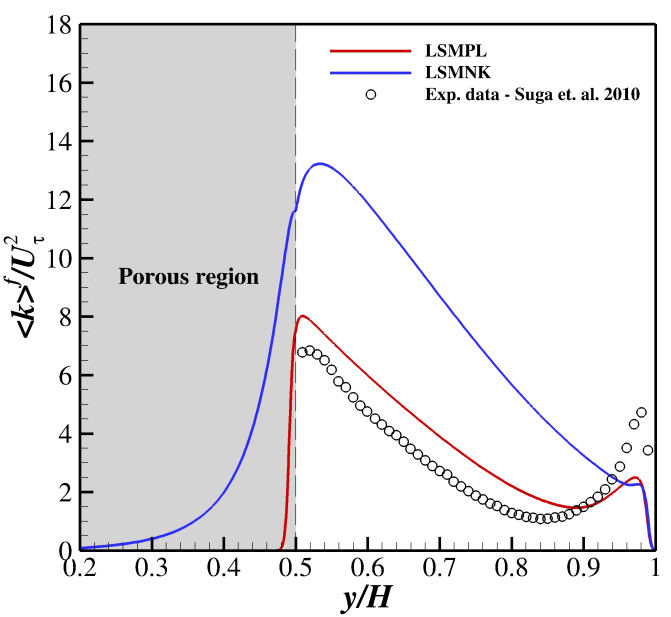

(b) Turbulent kinetic energy profile of Case\#13M

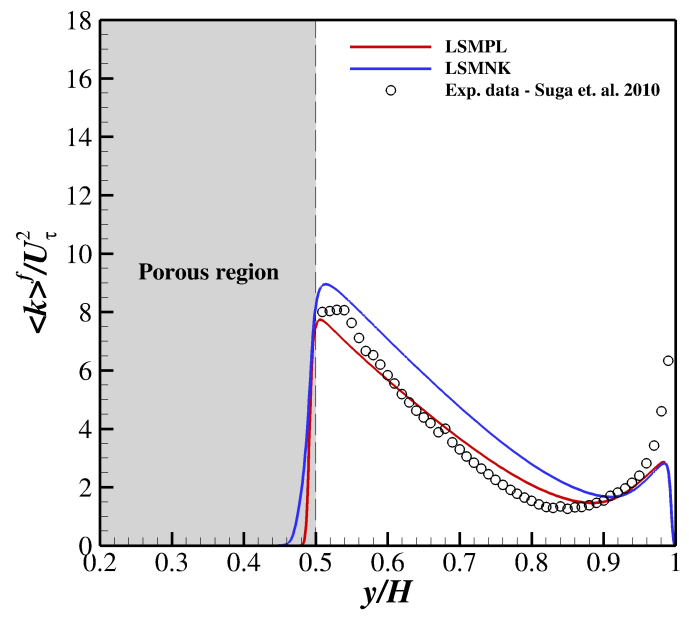

(d) Turbulent kinetic energy profile of Case\#13H

Fig. 3: Comparison between the current predictions and the experimental data Suga et al. (2010) of mean velocity and turbulent kinetic energy profiles in the \#13M and \#13H cases. 


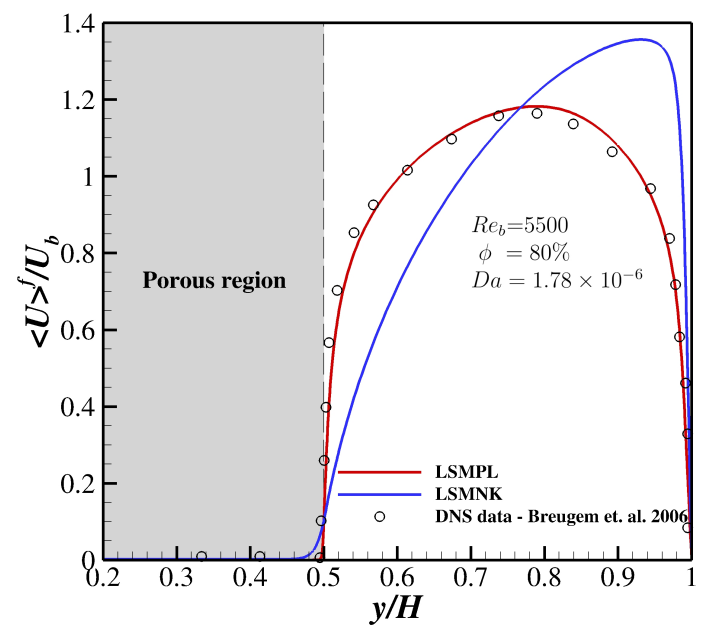

(a) Streamwise mean velocity profile

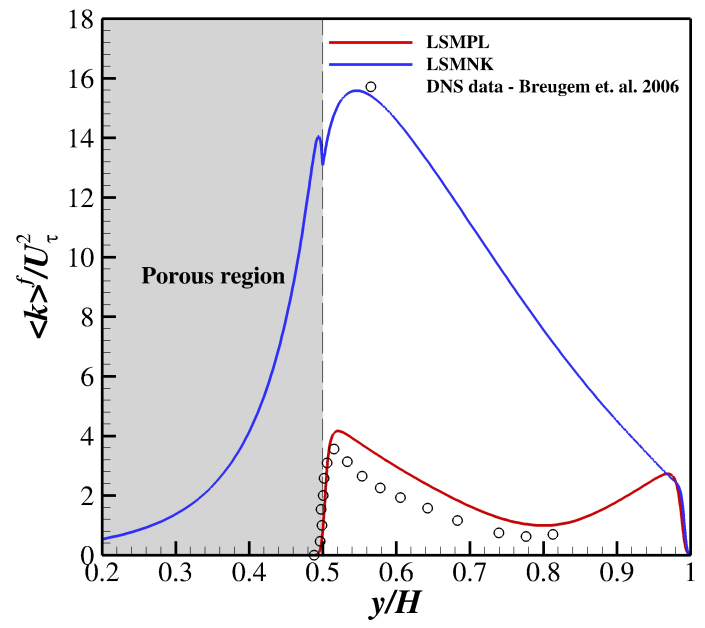

(b) Turbulent kinetic energy profile

Fig. 4: Comparison between the current predictions and the DNS data Breugem et al. (2006), case E80. 


\subsection{Turbulent Porous/Solid Rib Channel Flows}

For further validation for the modified model, flows along a channel again half-filled with a porous layer, but now with a solid (or porous) square rib, of half the clear channel height, mounted on top of this layer, as shown in Figure 5, have been considered. In the case of the porous rib, it is made of the same material as the porous layer beneath it. Whereas one would expect a significant flow separation to form behind a solid rib mounted on a solid surface, since fluid can now pass through the porous layer beneath the rib (and also though the rib itself in the porous rib case), this recirculation is either very much reduced, or not even present in some cases. Downstream of the rib, fluid can seep back into the clear fluid region, and the adverse pressure gradient that this sets up in the porous region can lead to a recirculation region forming there and in the near-interface part of the clear fluid region, as shown schematically in Figure 5a. The size of this bubble and its location are affected by the permeability of the porous layer (or both porous layer and rib in case of porous rib).

\subsubsection{Case description}

The cases considered have been studied experimentally by Suga et al. (2013). Both low, PPI=20, and high, $\mathrm{PPI}=6$, permeability of the porous layer (and the porous rib) are tested. For simplicity, the solid rib flow will be named by case \#PPI-s, and similarly, the porous rib cases are named as case\#PPI-p, as in Table 3 . The parameters of porous media that are used in the two cases are the same as those identified for cases \#20 and \#6 in the porous channel flows listed in Table 2.

To ensure sufficient upstream and downstream flow development lengths, the computational domain, as depicted in Figure $5 \mathrm{~b}$, extends from $12 h$ upstream of the porous rib to $51 h$ downstream of the rib. Fully developed flow profiles (from a separate calculation) are imposed at the inlet, with zero streamwise gradients applied at the outlet, and no-slip conditions at the impermeable walls. For the solid rib cases, a block-structured grid of around $1156(x) \times 297(y)$ cells was chosen, after grid independence tests. Similarly, for the porous rib cases, a grid of $368(x) \times 215(y)$ cells was found to be sufficient. The distribution of grid nodes was concentrated towards the porous-fluid interfaces and solid wall, as shown in Figure $5 \mathrm{c}$. The grid distribution in the streamwise direction for the solid rib case had to be much finer in the region behind the rib than in the porous rib cases, to capture the recirculation region present there in the former, which was largely not present in the porous rib cases.

Table 3: Characteristics of the solid/porous rib flow cases considered.

\begin{tabular}{lccccc}
\hline Rib & Case & $R e_{b}$ & $D a$ & $\phi$ & PPI \\
\hline Solid & $\# 20-\mathrm{s}$ & 10,000 & $6.2 \times 10^{-6}$ & $82 \%$ & 20 \\
& $\# 6-\mathrm{s}$ & 10,000 & $2.6 \times 10^{-5}$ & $80 \%$ & 6 \\
Porous & $\# 20-\mathrm{p}$ & 9,800 & $6.2 \times 10^{-6}$ & $82 \%$ & 20 \\
& $\# 6-\mathrm{p}$ & 10,600 & $2.6 \times 10^{-5}$ & $80 \%$ & 6 \\
\hline
\end{tabular}




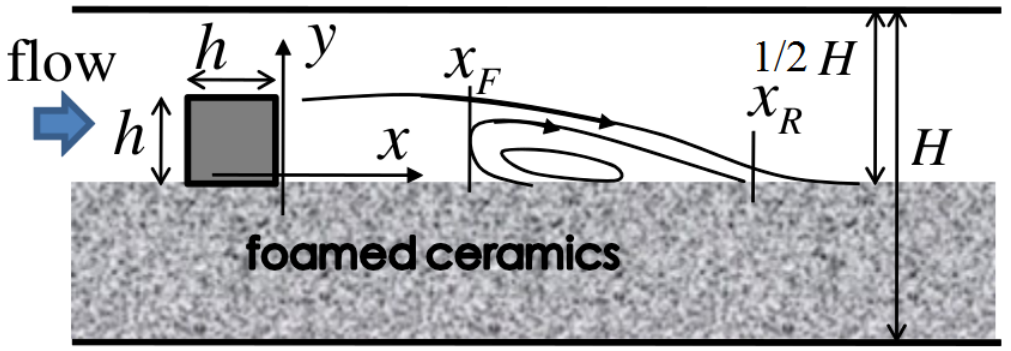

(a) Schematic of the circulating flow behind the rib with its front edge and reattachenment point.

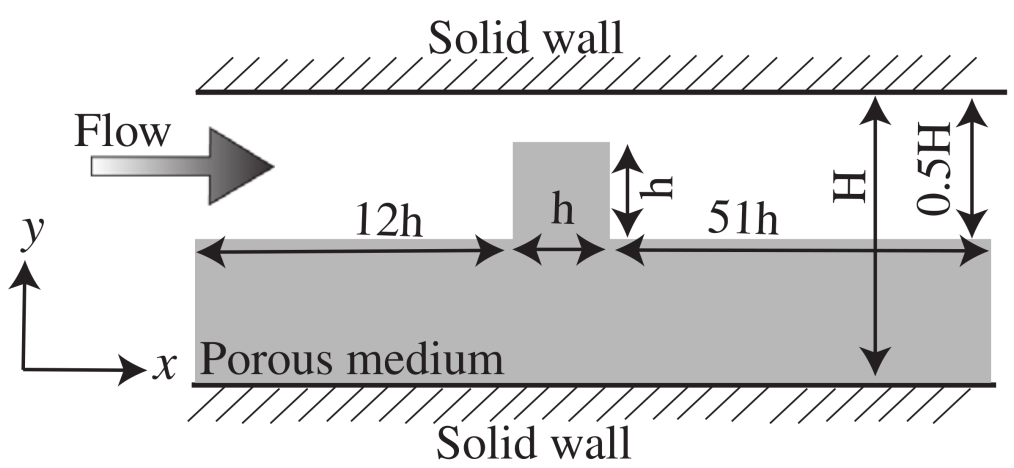

(b) Schematic of the computational domain

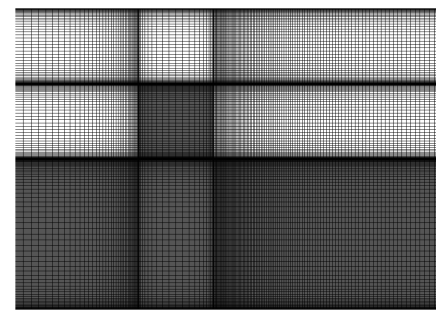

(c) Computational grid

Fig. 5: Configuration of computational domain and grid for a turbulent porous/solid rib channel flows.

\subsubsection{Results}

To provide a qualitative picture of the predicted flow features, Figure 6 shows the predicted streamlines, using the LSMNK scheme, for the two porosity cases, both with a solid and a porous rib. It can be clearly seen that, due to the resistance of the rib, some of the incoming fluid is directed towards the upper wall. However, the permeability of the lower channel layer permits a certain amount of fluid to pass through it, and in the case of the porous rib fluid can penetrate both the rib and the porous layer beneath it. In contrast to what would be seen in the case of a solid rib mounted on an impermeable surface, there is no flow separation or reversal upstream of the rib. The solid rib cases do show a small flow recirculation towards the leading edge of the top surface of the rib, as shown in Figures 6a and 6c although in the case of the porous rib this is not present, since some fluid does travel through the rib here. A low pressure region develops behind the rib, and this leads to fluid that has passed underneath the rib being entrained back into the clear fluid region behind the rib. As a consequence of this fluid movement there is only a small and weak separation and recirculation seen immediately behind the solid rib, and none behind the porous rib as fluid also passes through the rib itself in this case. As fluid continues to be entrained back into the clear fluid region downstream of the rib, a weak adverse pressure gradient is set up in the porous region. This leads to flow separation from the lower solid wall (seen at around $\mathrm{x} / \mathrm{h}=3.2$ in case\#20-s, for example), and a recirculation region forms across the porous region, extending slightly into the clear fluid region, as 
shown in Figure 6a. As the permeability increases, or the rib is made porous, fluid bleeds back into the clear channel more gradually, and the recirculation within the porous region is weaker and occurs further downstream. Such a recirculation feature was also deduced to be present, at least qualitatively, by Suga et al. (2013) in their experimental study, from analysing their measured mass flow rates in the clear fluid region and the streamwise pressure variation.

For a quantitative assessment of the results, Figures 7 and 8 show streamwise velocity profiles at a selection of streamwise locations for the flow over the solid and porous ribs respectively. It can be seen that the two present model predictions agree very well with each other and with the experimental data in all cases. In the porous rib case, the two present model predictions also agree with those obtained by Kuwata et al. (2014), as shown in Figure 8. As noted from the streamline figures, the velocity immediately behind the porous rib is higher than that behind the solid one (as a result of fluid passing through the rib). The figures also show that as the permeability is increased, the fluid velocity through the porous region underneath the rib (and through the rib itself in the porous rib cases) increases. Downstream of the rib, as the flow begins to relax back to its fully developed state, the velocity profiles show a highly asymmetric shape across the clear fluid region, in agreement with the measured data.

Corresponding profiles of turbulent kinetic energy and Reynolds shear stress for the four cases are shown in Figures 9, 10,11 and 12, It can be seen that high levels of turbulent kinetic energy generally occur close to the upstream edge of the rib, and then in the shear layers downstream, corresponding to the regions where the mean strains will result in significant turbulence generation. It should be noted that in Figures 9 and 10 the experimental turbulent kinetic energy values were estimated, based on $k=\frac{1}{2}\left[\overline{u^{2}}+\overline{v^{2}}+\left(\overline{u^{2}}+\bar{v}^{2}\right) / 2\right]$, where $u$ and $v$ are the fluctuating velocity components in the streamwise and wall-normal directions, respectively (Suga et al. 2013).

Examining the solid rib cases first, there is generally fairly good agreement between the predictions and experimental data in the clear fluid regions. However, both models tend to overpredict turbulence levels around the leading edge of the rib (as shown by $k$ profiles just above the rib at $x / h=-1$ and -0.5 , for example). Despite the high $k$ levels here, the Reynolds shear stress predictions are reasonable, with peak levels slightly underpredicted immediately downstream of the rib, and both $k$ and $\overline{u v}$ profiles show good agreement with the experimental data further downstream. The overprediction of $k$ around the leading edge of the rib is almost certainly due to the well-known failure of the underlying Launder-Sharma scheme (and most other linear EVM's) in impinging and stagnation flows, where the normal straining will give rise to erroneous Reynolds normal stresses, and consequently high levels of turbulence generation.

Turning to the porous rib cases, the overall level of agreement between the predictions and measurements in the clear-fluid region is again quite reasonable. The present predictions also agree very well with those obtained by Kuwata et al. (2014) using a multi-scale $k-\varepsilon$ model, as shown in Figure 10a. In the lower permeability case (Case\#20-p) there is again an overprediction of turbulent kinetic energy around the leading edge of the rib, although not as severe as in the solid rib case, since the impingement and normal straining is now weaker in this region as a result of some fluid being able to pass through the rib. In the higher permeability case (Case\#6-p), where the impingement is now even weaker, there is no noticeable overprediction of $k$ in this region. Further downstream, the peak $k$ levels tend to be slightly underpredicted in the lower permeability case (although the corresponding Reynolds shear stress peak levels are slightly overpredicted).

Within the porous layer beneath the rib (and within the rib itself, in the porous rib cases), there are quite different levels of turbulent kinetic energy predicted by the two models, with the LSMPL scheme generally returning significantly higher levels than the LSMNK. These differences are believed to be largely due the aforementioned behaviour of the underlying linear EVM in normally strained flow regions, coupled with the different forms of porosity-dependent source terms employed by the two schemes in their $k-\varepsilon$ equations. In the LSMPL scheme these source terms are dependent on both the superficial mean velocity and the turbulence quantities themselves, whereas in the LSMNK model they do not depend directly on the turbulence quantities. In regions of normal straining, where the linear EVM formulation tends to lead to 
overpredicted turbulence levels, these source terms therefore tend to be larger in the LSMPL scheme than in the LSMNK, leading to the higher $k$ levels seen in the figures. The same process is also believed to give rise to the higher levels of $k$ and $\overline{u v}$ seen in the LSMPL predictions further downstream in the porous layer for Case\#20 (after around $x / h=3$ for the solid rib case, and for $x / h>5$ in the porous rib case). In this case the normal straining is associated with the recirculation zone identified in this region when examining the streamline predictions. Although the recirculation, and associated normal straining, are quite weak here, so is the shearing, and hence the normal straining contribution to turbulence generation is still significant. There are no direct measurements within the porous region, but the $k$ data near the interface in the clear fluid region suggests that the LSMPL scheme tends to overpredict turbulence levels in these parts of the porous region (particularly for $x / h>6$ in the Case\#20-p, for example). In the higher permeability case (Case\#6-p) the weaker recirculation is not seen to have a significant influence on the predicted turbulence levels in this region. 


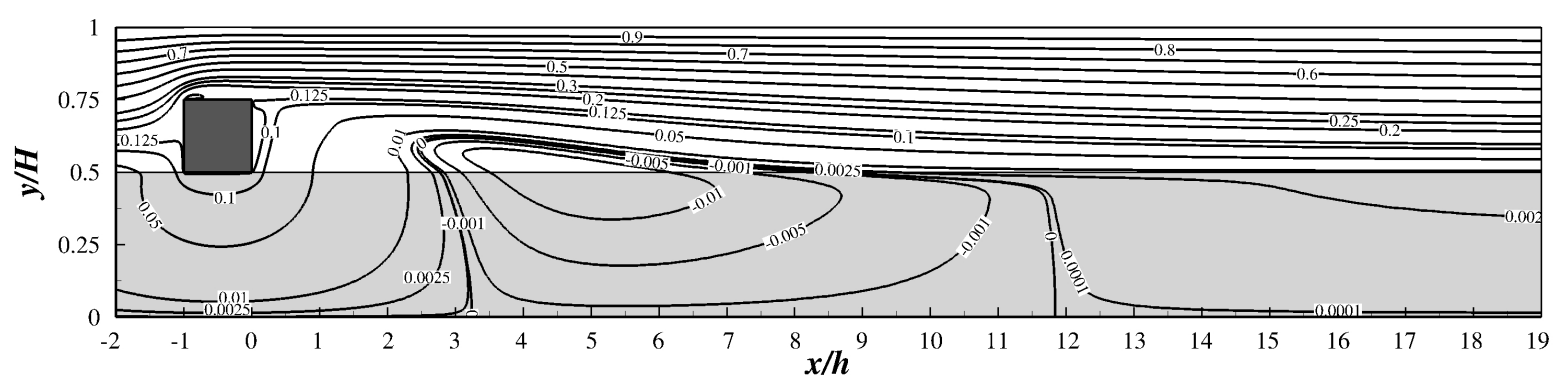

(a) Case \#20-s, $\left(\mathrm{Da}=6.2 \times 10^{-6}, \mathrm{PPI}=20\right)$

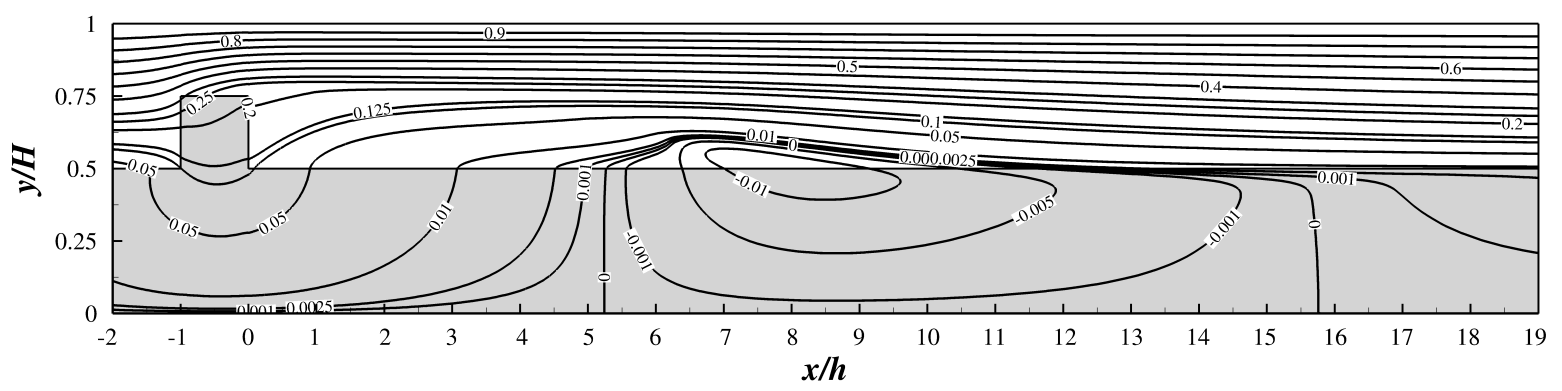

(b) Case\#20 $-p,\left(\mathrm{Da}=6.2 \times 10^{-6}, \mathrm{PPI}=20\right)$

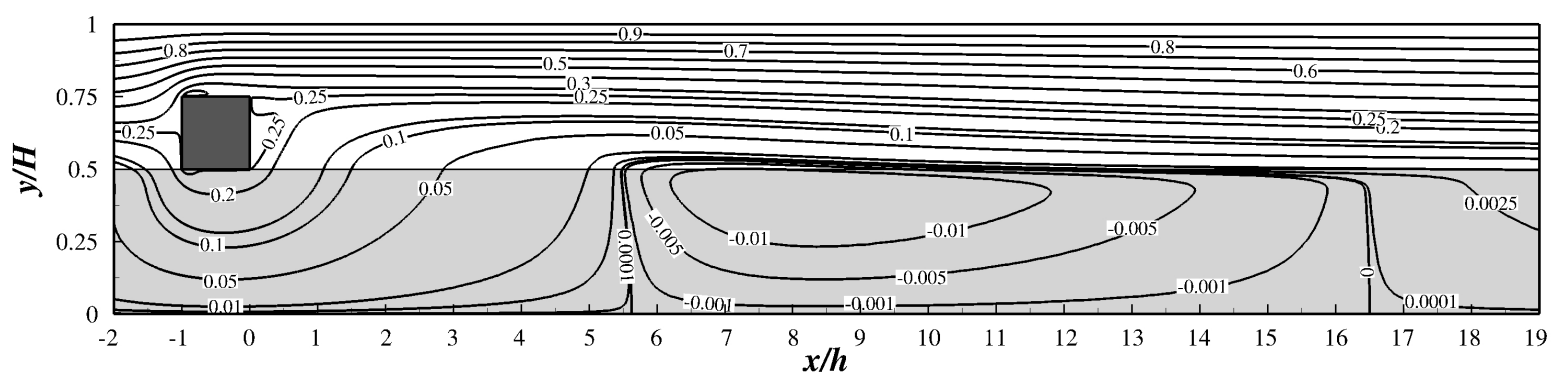

(c) Case\#6 $-s,\left(\mathrm{Da}=2.6 \times 10^{-5}, \mathrm{PPI}=6\right)$

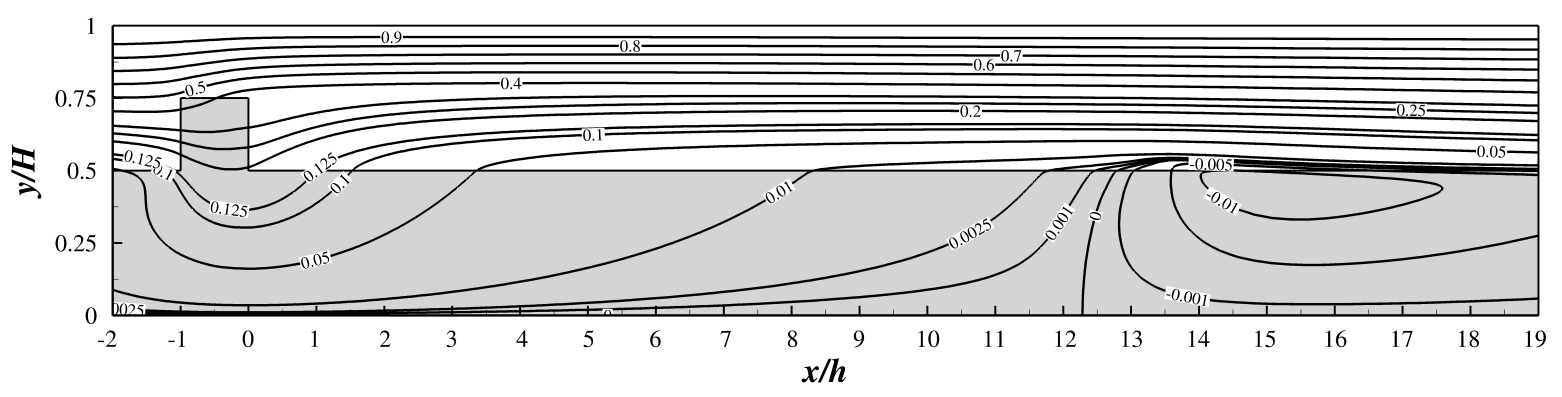

(d) Case\#6 $-p,\left(\mathrm{Da}=2.6 \times 10^{-5}, \mathrm{PPI}=6\right)$

Fig. 6: Predicted streamlines, LSMNK model 


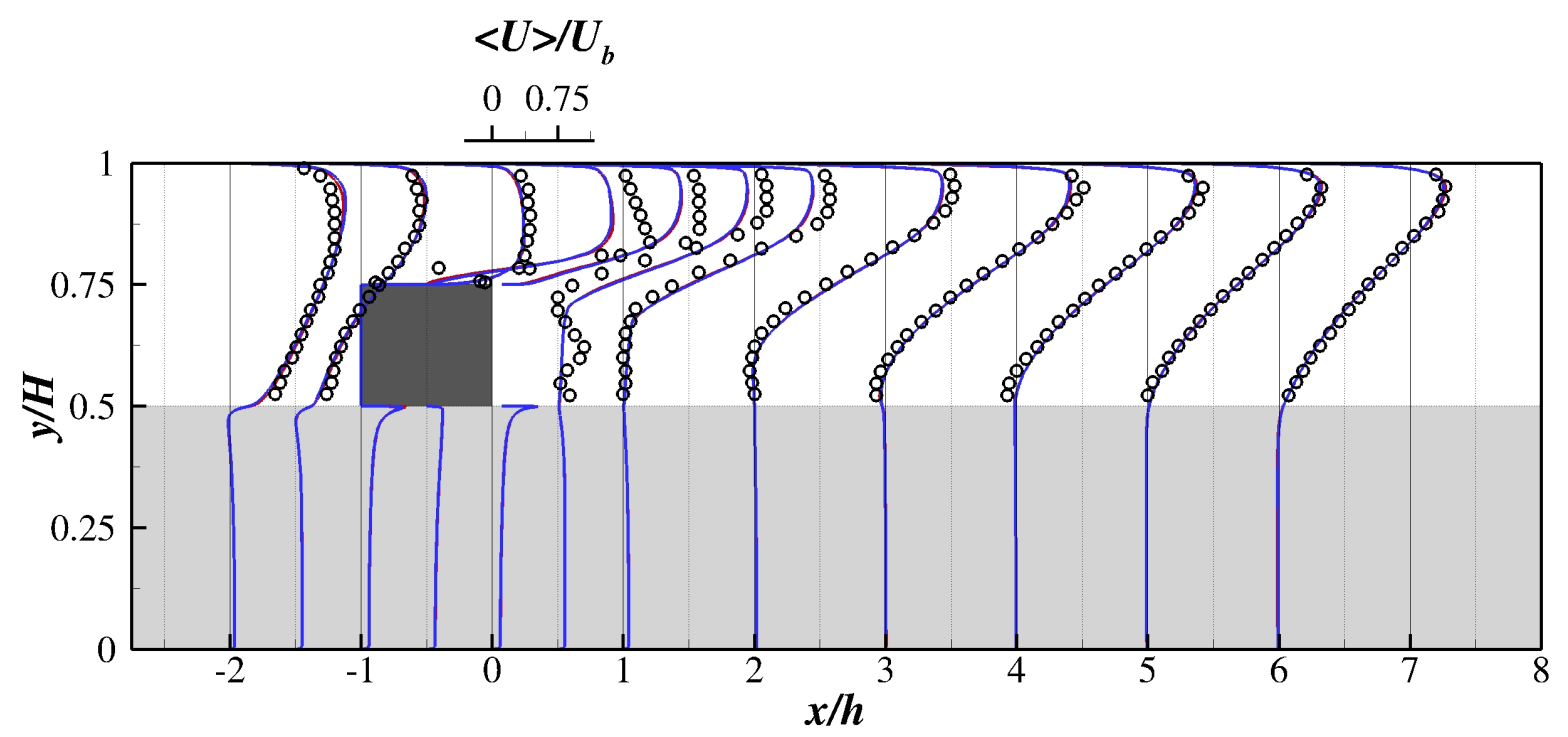

(a) Case $\# 20-s,\left(\mathrm{Da}=6.2 \times 10^{-6}, \mathrm{PPI}=20\right)$

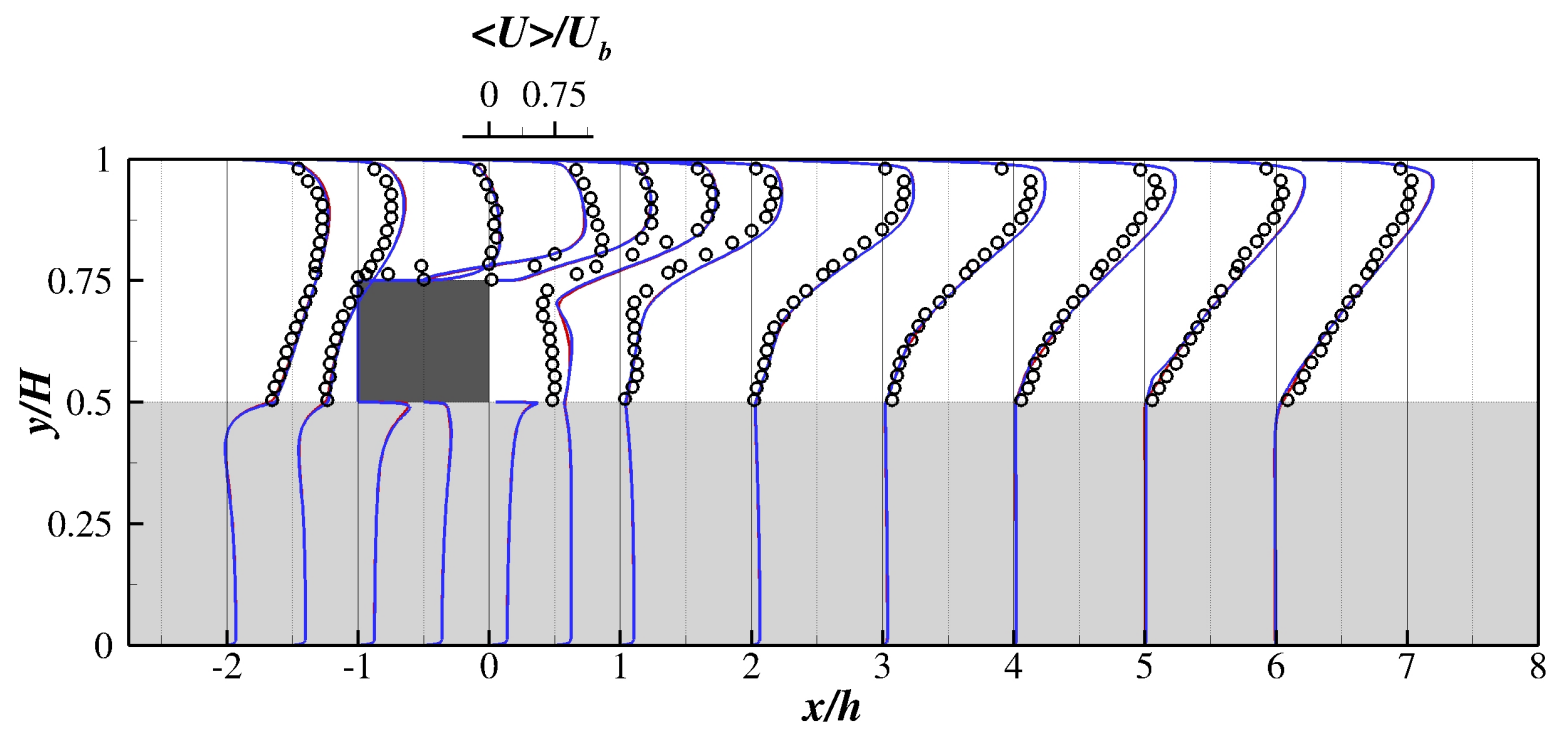

(b) Case\#6 $-s,\left(\mathrm{Da}=2.6 \times 10^{-5}, \mathrm{PPI}=6\right)$

Fig. 7: Comparison for streamwise velocity profiles between the current predictions for the low permeability and the experimental data Suga et al. (2013) for turbulent porous channel flows with mounted solid rib, (Red lines represent the LSMPL model, blue lines represent the LSMNK model and symbols represent experimental data) 


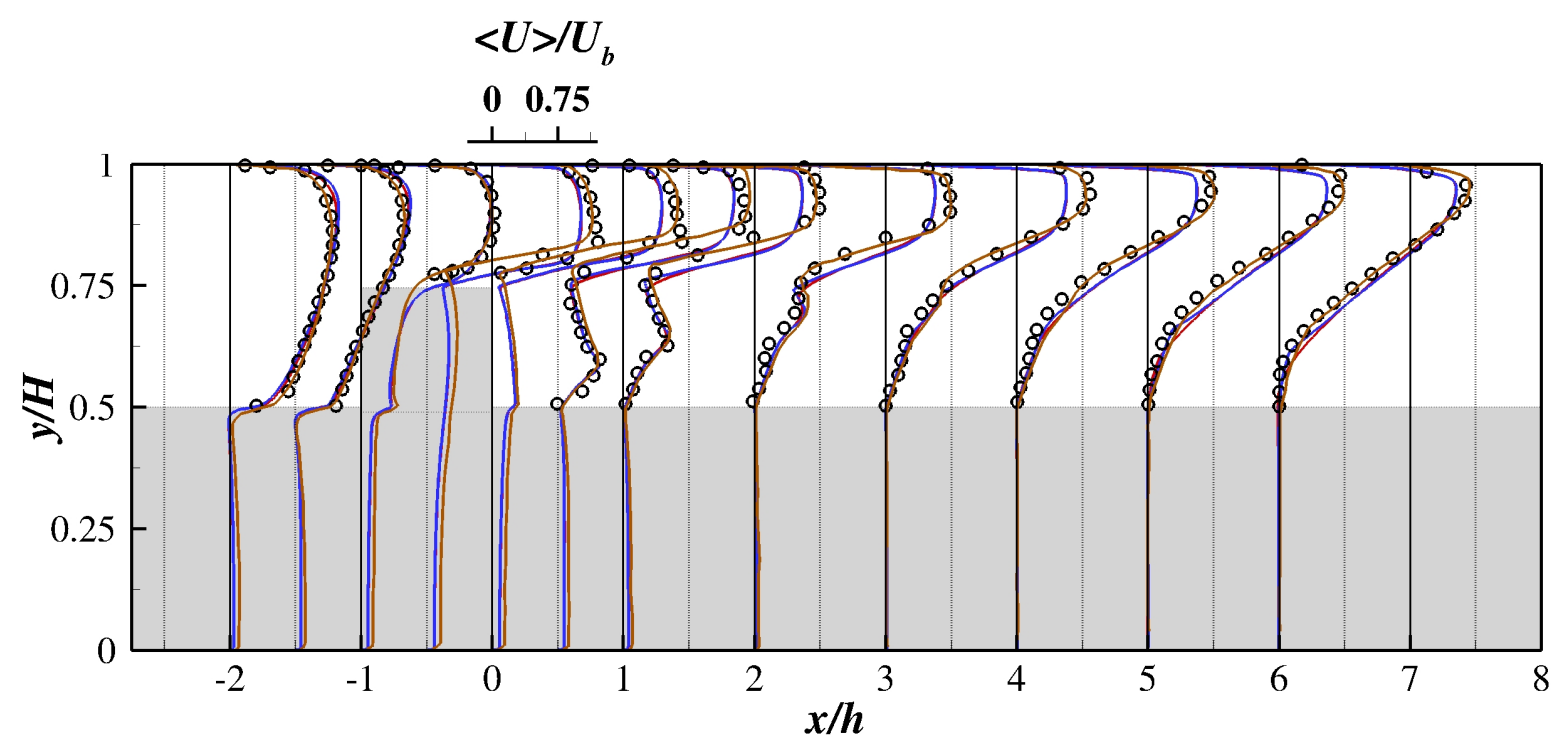

(a) Case\#20 - p, $\left(\mathrm{Da}=6.2 \times 10^{-6}, \mathrm{PPI}=20\right)$

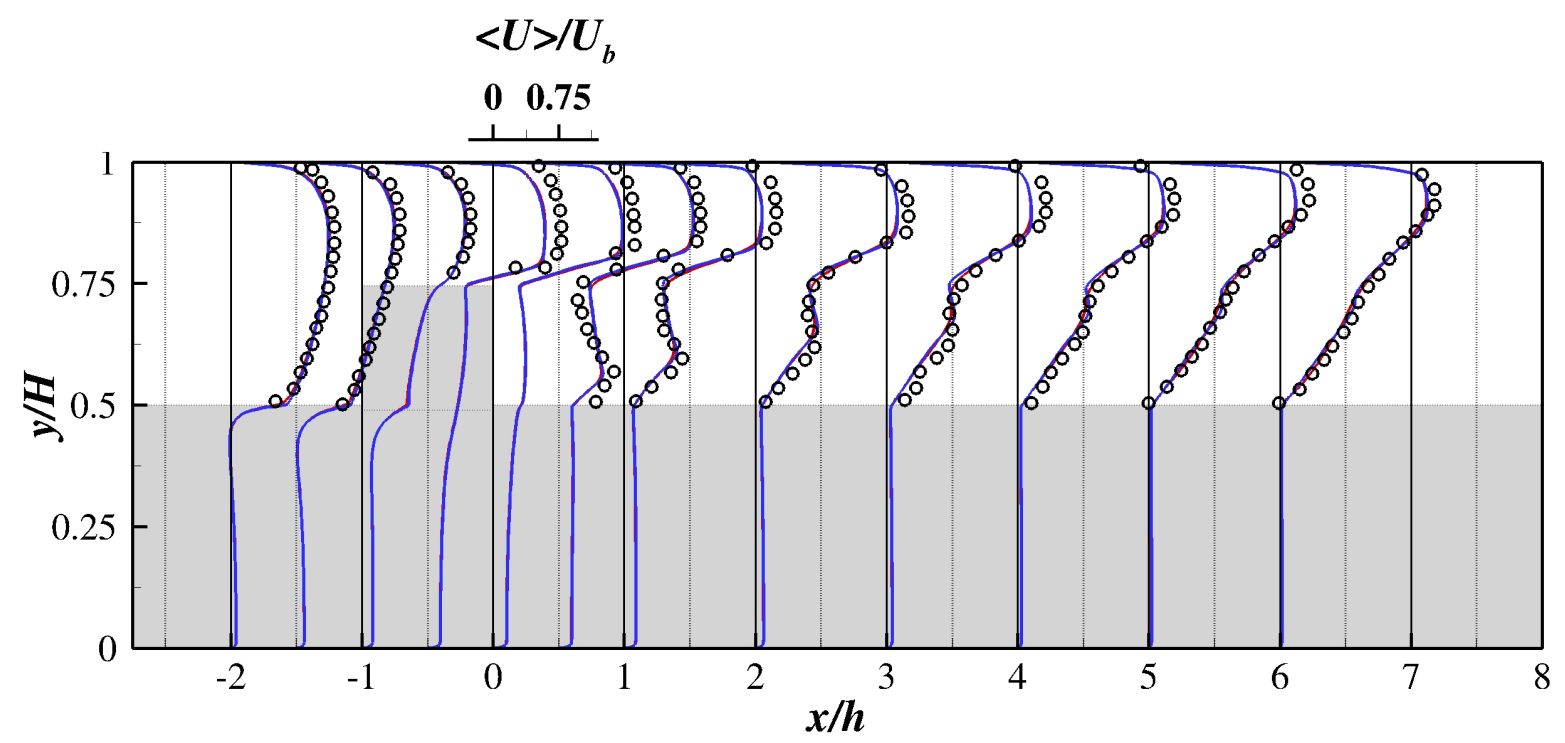

(b) Case\#6 $-p,\left(\mathrm{Da}=2.6 \times 10^{-5}, \mathrm{PPI}=6\right)$

Fig. 8: Comparison for streamwise velocity profiles for the current predictions with both the experimental data Suga et al. (2013) and the calculations of Kuwata et al. (2014) for turbulent porous channel flows with mounted porous rib, (Red lines represent the LSMPL model, blue lines represent the LSMNK model, symbols represent experimental data and brown lines are calculations of Kuwata et al. (2014)). 


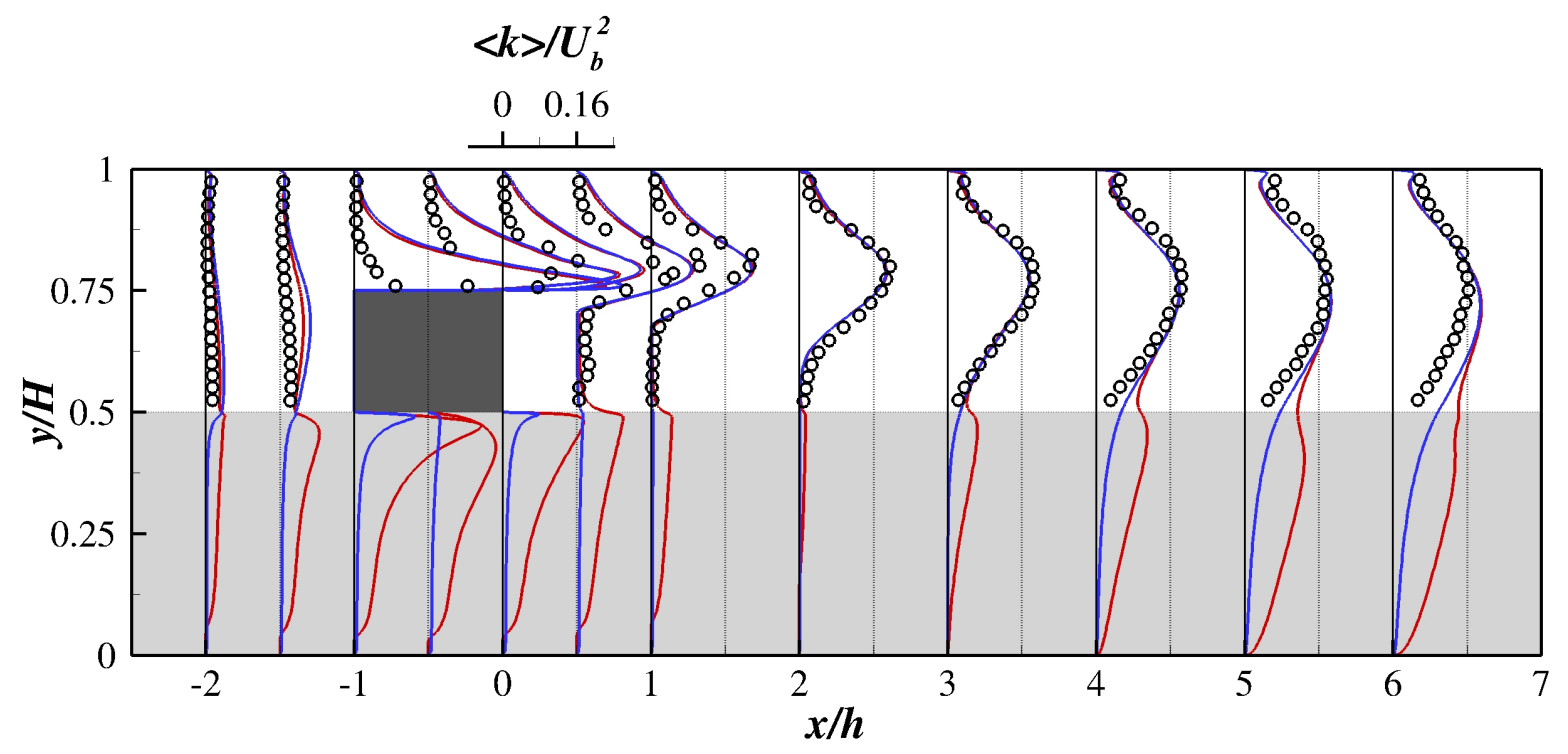

(a) Case\#20 - s, $\left(\mathrm{Da}=6.2 \times 10^{-6}, \mathrm{PPI}=20\right)$

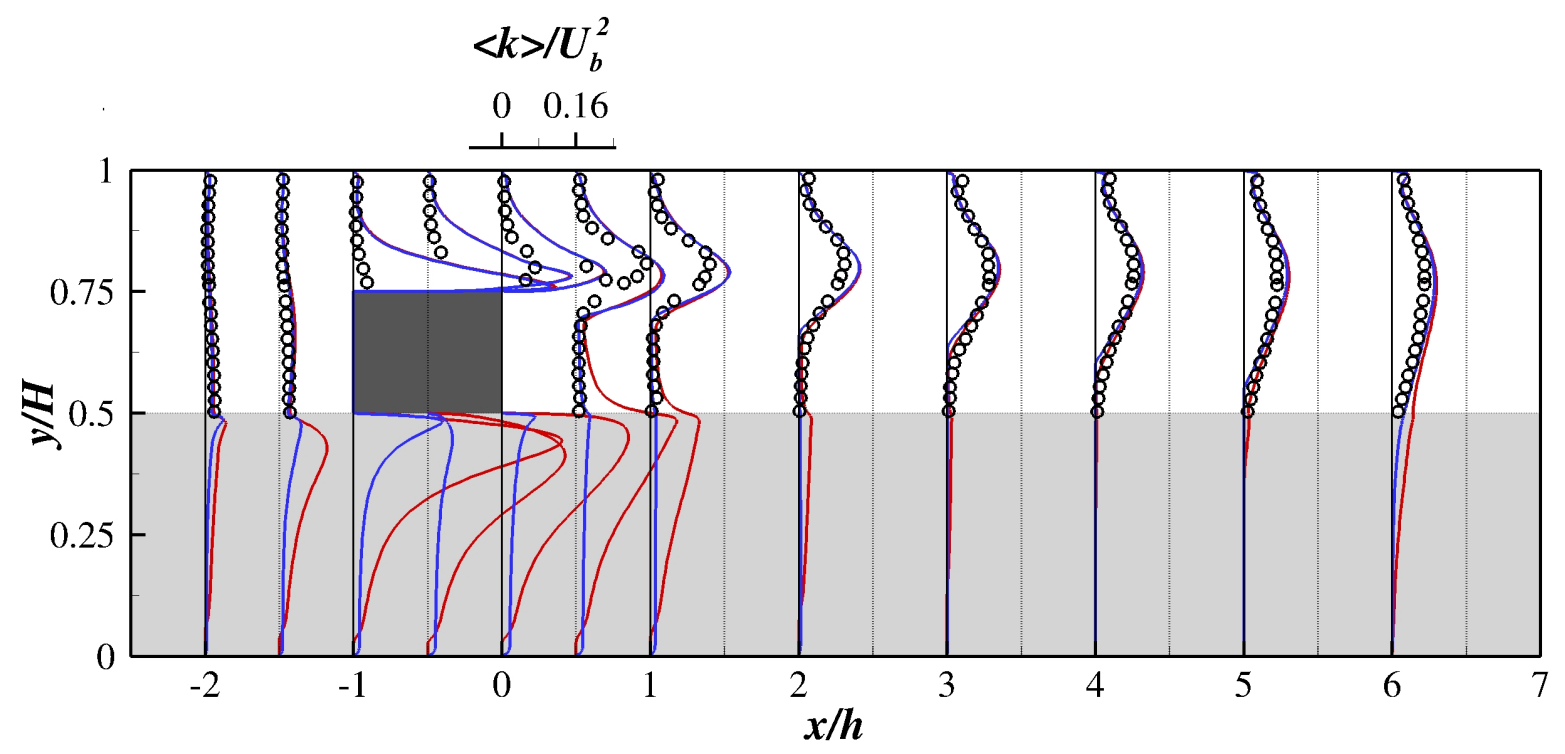

(b) Case\#6 $-s,\left(\mathrm{Da}=2.6 \times 10^{-5}, \mathrm{PPI}=6\right)$

Fig. 9: Comparison for turbulent kinetic energy profiles between the current predictions for the low permeability and the experimental data Suga et al. (2013) for turbulent porous channel flows with mounted solid rib, (Red lines represent the LSMPL model, blue lines represent the LSMNK model and symbols represent experimental data). 


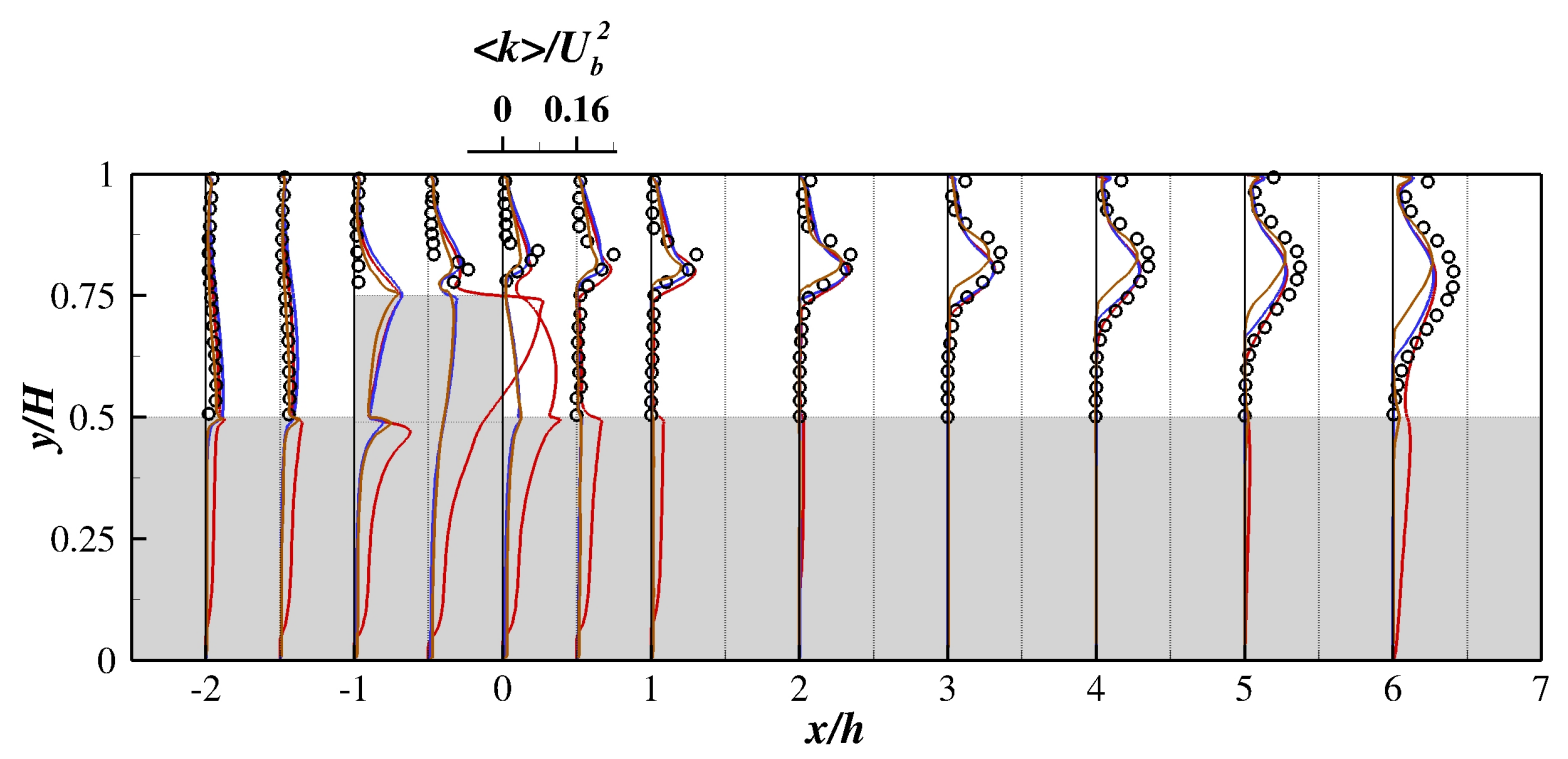

(a) Case $\# 20-p,\left(\mathrm{Da}=6.2 \times 10^{-6}, \mathrm{PPI}=20\right)$.

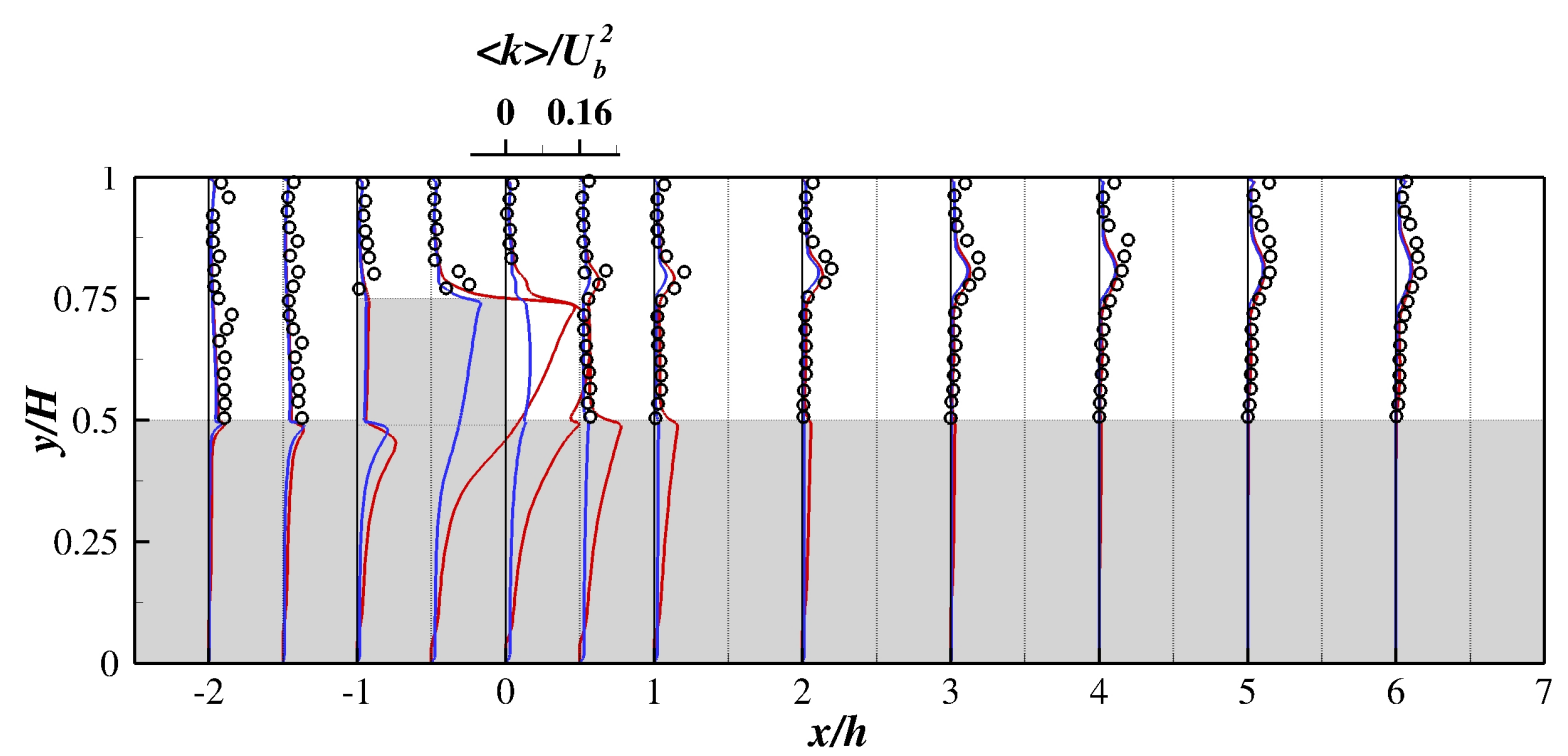

(b) Case\#6 $-p,\left(\mathrm{Da}=2.6 \times 10^{-5}, \mathrm{PPI}=6\right)$

Fig. 10: Comparison for turbulent kinetic energy profiles with both the experimental data Suga et al. (2013) and the calculations of Kuwata et al. (2014) for turbulent porous channel flows with mounted porous rib, (Red lines represent the LSMPL model, blue lines represent the LSMNK model, symbols represent experimental data and brown lines are calculations of Kuwata et al. (2014)). 


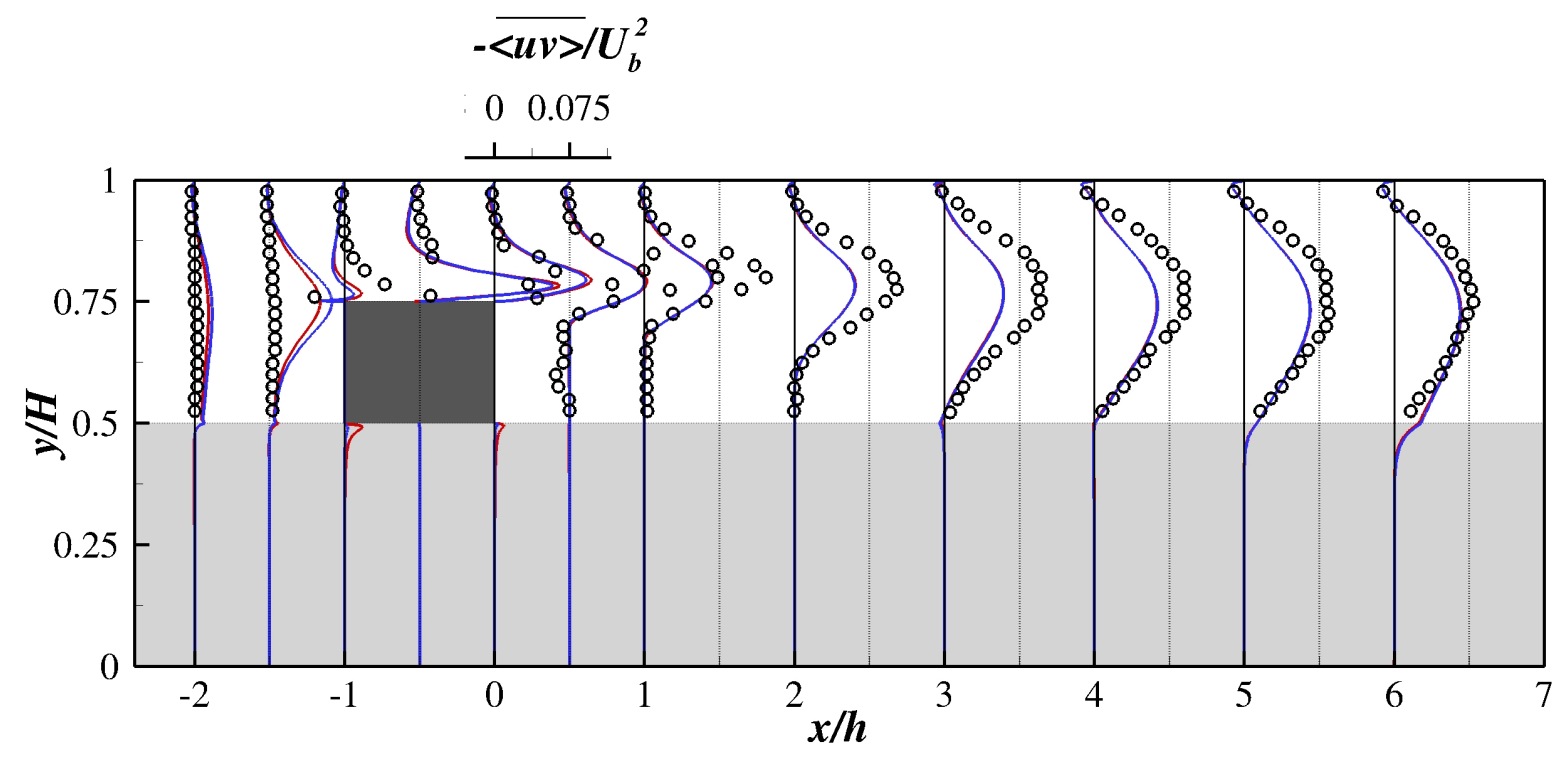

(a) Case\#20-s, (Da $\left.=6.2 \times 10^{-6}, \mathrm{PPI}=20\right)$

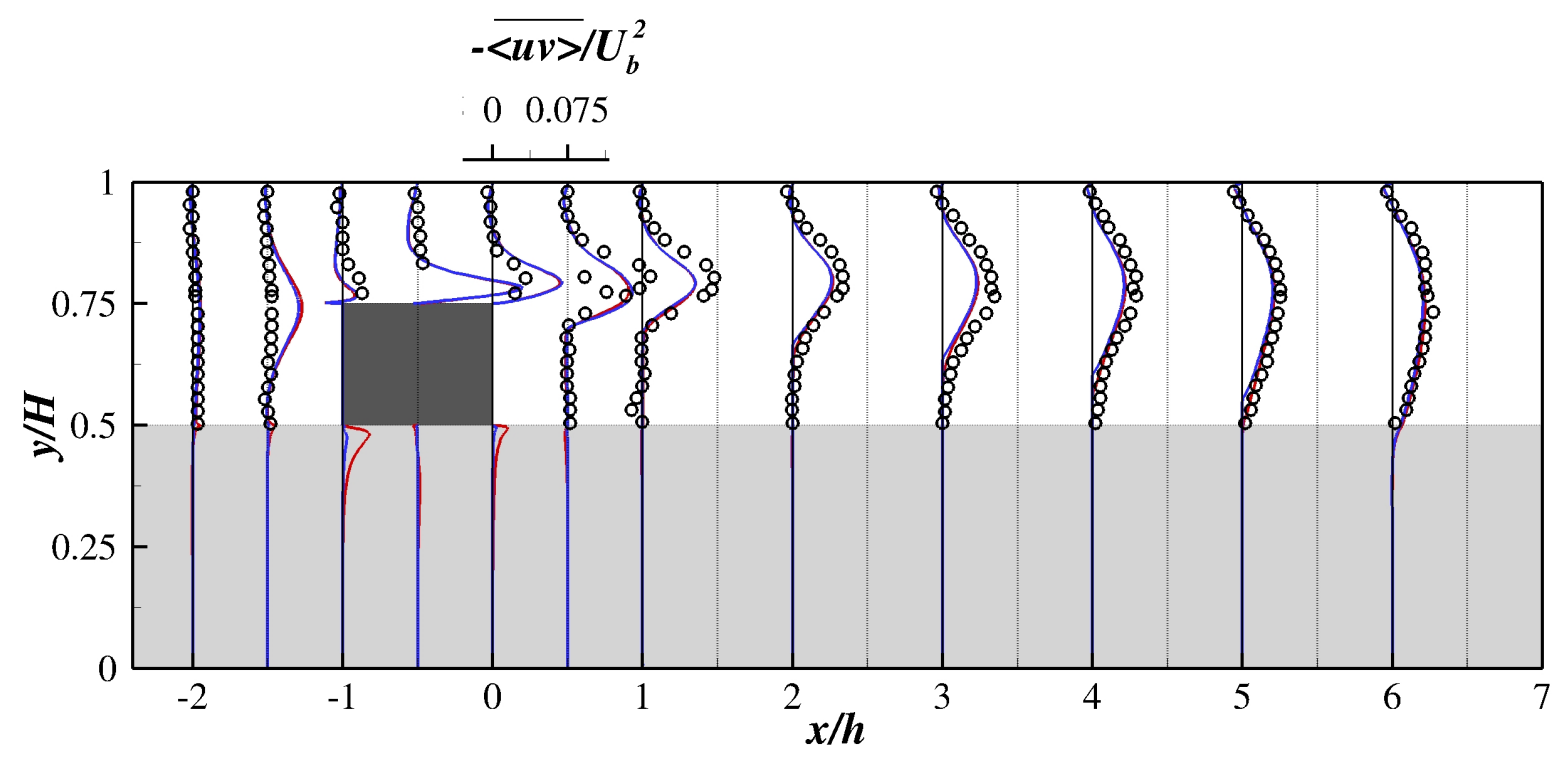

(b) Case\#6 $-s,\left(\mathrm{Da}=2.6 \times 10^{-5}, \mathrm{PPI}=6\right)$

Fig. 11: Comparison for Reynolds shear stress profiles between the current predictions for the low permeability and the experimental data Suga et al. (2013) for turbulent porous channel flows with mounted solid rib, (Red lines represent the LSMPL model, blue lines represent the LSMNK model and symbols represent experimental data). 


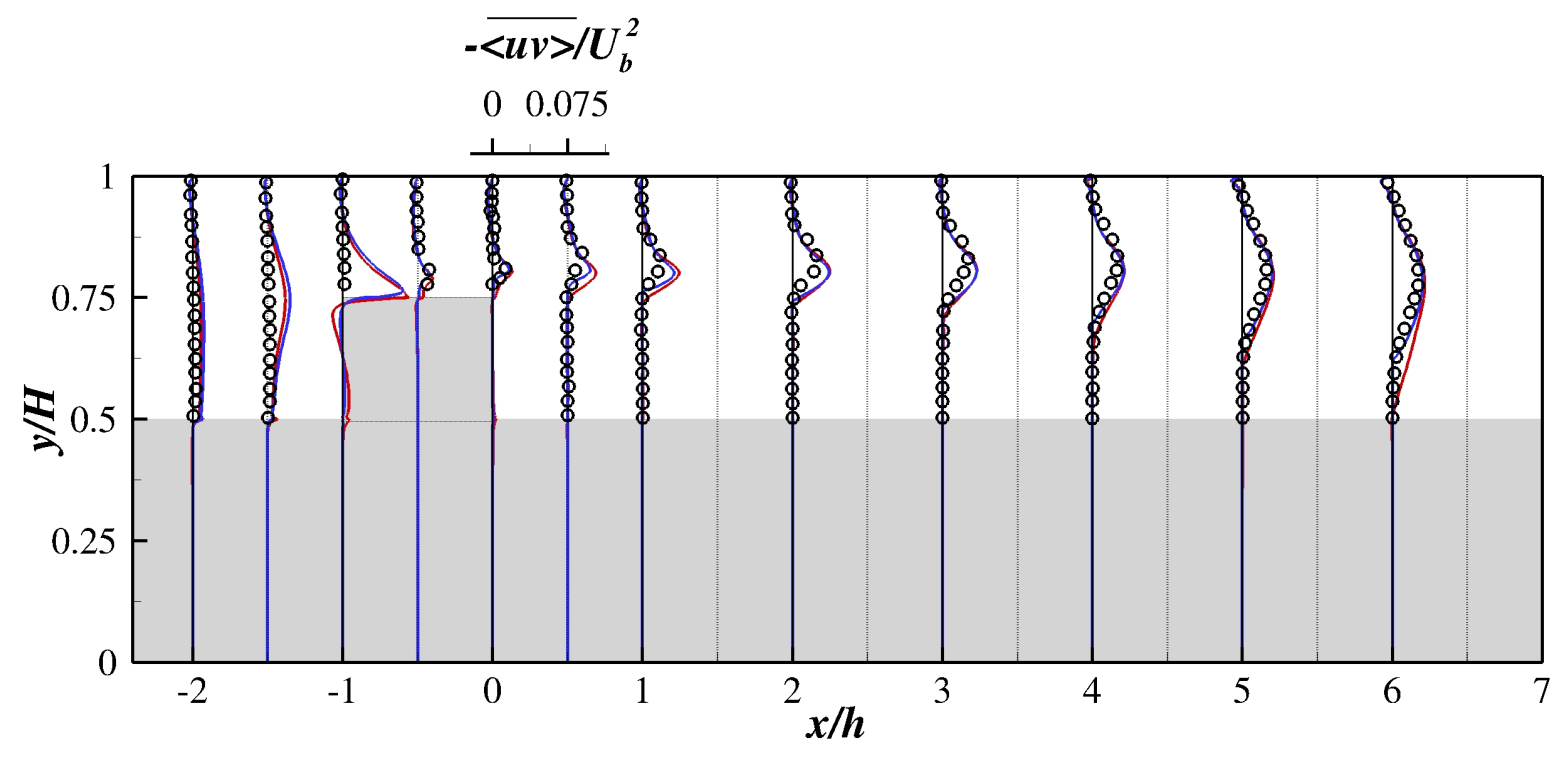

(a) Case\#20 - p, $\left(\mathrm{Da}=6.2 \times 10^{-6}, \mathrm{PPI}=20\right)$

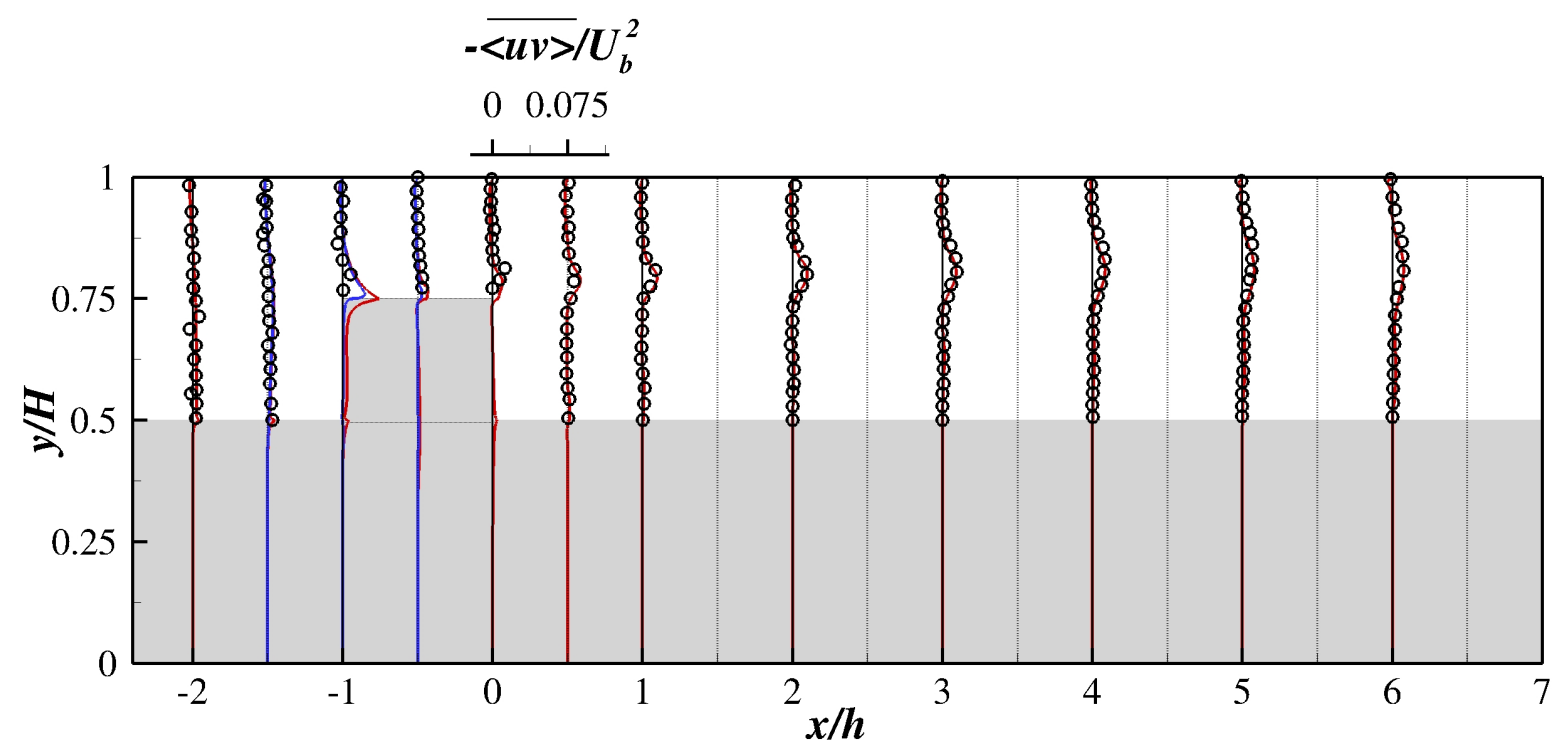

(b) Case\#6 $-p,\left(\mathrm{Da}=2.6 \times 10^{-5}, \mathrm{PPI}=6\right)$

Fig. 12: Comparison for Reynolds shear stress profiles between the current predictions for the high permeability and the experimental data Suga et al. (2013) for turbulent porous channel flows with mounted porous rib, (Red lines represent the LSMPL model, blue lines represent the LSMNK model and symbols represent experimental data). 


\section{Conclusions}

Two different modifications of the Launder-Sharma model, those proposed by Nakayama and Kuwahara (2008) and Pedras and de Lemos (2001b) have been tested, representing widely-used schemes for turbulent flow in porous media applications. These have been combined with near-interface damping terms for the modelled porous source terms, which have been initially proposed by Kuwata and Suga (2013) for secondmoment closures and which in the course of this study have been re-optimized for the $k-\varepsilon$ model. The main contribution of this research is that the proposed interface-damping terms have been shown to improve significantly the predictions around the porous/clear fluid interface region. The results of the turbulent porous channel flows show that the prediction accuracy of both modified models is generally satisfactory, although at relatively low Reynolds numbers and low permeability the LSMNK results become poorer, with turbulence levels quite significantly overpredicted. This is attributed to a weakness of the source terms of the LSMNK model at quite low Reynolds numbers, due to a greater sensitivity to the permeability of porous media.

For the solid/porous rib channel flow cases, the results indicated that the predictions of the current modified models were in a satisfactory agreement with the experimental data reported by Suga et al. (2013) for both the solid and porous ribs. The results showed that the recirculation or wake flow formed behind the rib weakened and was moved downstream, compared to the location of the recirculation region for flow behind a solid rib mounted on an impermeable surface.

The turbulence levels around and behind the rib were affected by permeability. In the solid rib flows, as the permeability increased, the strength of the reverse flow behind the solid rib was reduced. This in turn damped the turbulent kinetic energy and Reynolds shear stress around and behind the rib. This was attributed to the reduction in the production of turbulent kinetic energy as a result of the lower velocity gradients.

In the porous rib flows, due to permeability of the rib, the entrained fluid penetrated the rib and the porous layer. Consequently, a lower pressure drop was seen between the upstream and downstream faces of the rib, which caused weaker reverse flow than that seen behind the solid rib. Therefore, lower levels of turbulent kinetic energy and Reynolds shear stress than in the solid rib case were also observed. In the lower permeability case both models tend to overpredict turbulence levels around the impingement region at the front of the solid/porous rib, as a result of the underlying weakness in the linear eddy-viscosity formulation in such flows. As a result of that, and due to the dependency of the source terms of the LSMPL model on the turbulent quantities, higher turbulence levels are returned in the LSMPL model than the LSMNK model within and beneath the rib. The LSMPL scheme also predicts higher near interface turbulence levels further downstream, where a weak recirculation occurs, for similar reasons.

The proposed model developments therefore lead to variants of a low-Re $k-\varepsilon$ model that show considerable promise in the simulation of turbulent flows through mixed porous and clear regions. This is highly relevant to the development of potential applications of porous materials. Further research will focus on the extension of these ideas to the transfer of thermal energy.

\section{Acknowledgements}

The financial support from the Ministry of Higher Education and Scientific Research of Iraq and the University of Kufa is gratefully acknowledged. The authors would like to acknowledge the assistance given by IT Services and the use of the Computational Shared Facility at The University of Manchester. 


\section{References}

Antohe, B. and Lage, J. (1997). A general two-equation macroscopic turbulence model for incompressible flow in porous media. International Journal of Heat and Mass Transfer, 40(13):3013-3024.

Beavers, G. and Joseph, D. (1967). Boundary conditions at a naturally permeable wall. Journal of fluid mechanics, 30(01):197-207.

Breugem, W., Boersma, B., and Uittenbogaard, R. (2006). The influence of wall permeability on turbulent channel flow. Journal of Fluid Mechanics, 562:35-72.

Chan, H., Zhang, Y., Leu, J., and Chen, Y. (2010). Numerical calculation of turbulent channel flow with porous ribs. Journal of Mechanics, 26(1):15-28.

Chan, H.-C., Huang, W., Leu, J.-M., and Lai, C.-J. (2007). Macroscopic modeling of turbulent flow over a porous medium. International Journal of Heat and Fluid Flow, 28(5):1157-1166.

Chandesris, M. and Jamet, D. (2006). Boundary conditions at a planar fluid-porous interface for a poiseuille flow. International Journal of Heat and Mass Transfer, 49(13-14):2137-2150.

Chandesris, M. and Jamet, D. (2009). Derivation of jump conditions for the turbulence $k-\varepsilon$ model at a fluid/porous interface. International Journal of Heat and Fluid Flow, 30(2):306-318.

De Lemos, M. J. (2005). Turbulent kinetic energy distribution across the interface between a porous medium and a clear region. International communications in heat and mass transfer, 32(1-2):107-115.

De Lemos, M. J. (2009). Turbulent flow around fluid-porous interfaces computed with a diffusion-jump model for k and $\varepsilon$ transport equations. Transport in porous media, 78(3):331-346.

Dunn, C., Lopez, F., and Garcia, M. H. (1996). Mean flow and turbulence in a laboratory channel with simulated vegatation (hes 51). Technical report.

Dybbs, A. and Edwards, R. (1984). A new look at porous media fluid mechanics-darcy to turbulent. In: Fundamentals of transport phenomena in porous media, pages 199-256. Springer, Dordrecht.

Finnigan, J. (2000). Turbulence in plant canopies. Annual review of fluid mechanics, 32(1):519-571.

Getachew, D., Minkowycz, W., and Lage, J. (2000). A modified form of the $k-\varepsilon$ model for turbulent flows of an incompressible fluid in porous media. International Journal of Heat and Mass Transfer, 43(16):2909-2915.

Hwang, J. (1998). Heat transfer-friction characteristic comparison in rectangular ducts with slit and solid ribs mounted on one wall. Transactions-American Society of Mechanical Engineers Journal of Heat Transfer, 120:709-716.

Iacovides, H. and Launder, B. (2007). Internal blade cooling: the cinderella of computational and experimental fluid dynamics research in gas turbines. Proceedings of the Institution of Mechanical Engineers, Part A: Journal of Power and Energy, 221(3):265-290.

Iacovides, H. and Raisee, M. (1999). Recent progress in the computation of flow and heat transfer in internal cooling passages of turbine blades. International Journal of Heat and Fluid Flow, 20(3):320-328.

Kaviany, M. (1991). Principles of heat transfer in porous media. Springer Science \& Business Media.

Kuwata, Y. and Suga, K. (2013). Modelling turbulence around and inside porous media based on the second moment closure. International Journal of Heat and Fluid Flow, 43:35-51.

Kuwata, Y., Suga, K., and Sakurai, Y. (2014). Development and application of a multi-scale $k-\varepsilon$ model for turbulent porous medium flows. International Journal of Heat and Fluid Flow, 49:135-150.

Kuznetsov, A. (2004). Numerical modeling of turbulent flow in a composite porous/fluid duct utilizing a two-layer k- $\varepsilon$ model to account for interface roughness. International journal of thermal sciences, 43(11):1047-1056.

Kuznetsov, A. and Xiong, M. (2003). Development of an engineering approach to computations of turbulent flows in composite porous/fluid domains. International journal of thermal sciences, 42(10):913-919.

Launder, B. and Sharma, B. (1974). Application of the energy-dissipation model of turbulence to the calculation of flow near a spinning disc. Letters in heat and mass transfer, 1(2):131-137.

Lee, K. and Howell, J. (1987). Forced convective and radiative transfer within a highly porous layer exposed to a turbulent external flow field. In Proceedings of the 1987 ASME-JSME Thermal Engineering Joint Conf, volume 2, pages $377-386$.

Leu, J., Chan, H., and Chu, M. (2008). Comparison of turbulent flow over solid and porous structures mounted on the bottom of a rectangular channel. Flow Measurement and Instrumentation, 19(6):331-337.

Lien, F. and Leschziner, M. (1994a). Multigrid acceleration for recirculating laminar and turbulent flows computed with a non-orthogonal, collocated finite-volume scheme. Computer Methods in Applied Mechanics and Engineering, 118(34):351-371.

Lien, F. and Leschziner, M. (1994b). Upstream monotonic interpolation for scalar transport with application to complex turbulent flows. International Journal for Numerical Methods in Fluids, 19(6):527-548.

Liou, T., Chen, S., and Shih, K. (2002). Numerical simulation of turbulent flow field and heat transfer in a two-dimensional channel with periodic slit ribs. International journal of heat and mass transfer, 45(22):4493-4505.

Masuoka, T. and Takatsu, Y. (1996). Turbulence model for flow through porous media. International Journal of Heat and Mass Transfer, 39(13):2803-2809.

Mößner, M. and Radespiel, R. (2015). Modelling of turbulent flow over porous media using a volume averaging approach and a reynolds stress model. Computers \& Fluids, 108:25-42. 
Naaktgeboren, C., Krueger, P., and Lage, J. (2012). Inlet and outlet pressure-drop effects on the determination of permeability and form coefficient of a porous medium. Journal of Fluids Engineering, 134(5):051209.

Nakayama, A. and Kuwahara, F. (1999). A macroscopic turbulence model for flow in a porous medium. TransactionsAmerican Society of Mechanical Engineers Journal of Fluids Engineering, 121:427-433.

Nakayama, A. and Kuwahara, F. (2008). A general macroscopic turbulence model for flows in packed beds, channels, pipes, and rod bundles. Journal of Fluids Engineering, 130(10):101205.

Nezu, I. and Sanjou, M. (2008). Turburence structure and coherent motion in vegetated canopy open-channel flows. Journal of hydro-environment research, 2(2):62-90.

Nikora, V., McEwan, I., M., S., C., S., Pokrajac, D., and Walters, R. (2007). Double-averaging concept for rough-bed open-channel and overland flows: Theoretical background. Journal of hydraulic Engineering, 133(8):873-883.

Nuntadusit, C., Wae-Hayee, M., Bunyajitradulya, A., and Eiamsa-ard, S. (2012). Thermal visualization on surface with transverse perforated ribs. International Communications in Heat and Mass Transfer, 39(5):634-639.

Ochoa-Tapia, J. and Whitaker, S. (1995). Momentum transfer at the boundary between a porous medium and a homogeneous fluid - i. theoretical development. International Journal of Heat and Mass Transfer, 38(14):2635-2646.

Panigrahi, P., Schröder, A., and Kompenhans, J. (2006). PIV investigation of flow behind surface mounted permeable ribs. Experiments in fluids, 40(2):277-300.

Panigrahi, P., Schröder, A., and Kompenhans, J. (2008). Turbulent structures and budgets behind permeable ribs. Experimental Thermal and Fluid Science, 32(4):1011-1033.

Patankar, S. and Spalding, D. (1972). A calculation procedure for heat, mass and momentum transfer in three-dimensional parabolic flows. International journal of heat and mass transfer, 15(10):1787-1806.

Pedras, M. and de Lemos, M. (2000). On the definition of turbulent kinetic energy for flow in porous media. International communications in heat and mass transfer, 27(2):211-220.

Pedras, M. and de Lemos, M. (2001a). Macroscopic turbulence modeling for incompressible flow through undeformable porous media. International journal of heat and mass transfer, 44(6):1081-1093.

Pedras, M. and de Lemos, M. (2001b). Simulation of turbulent flow in porous media using a spatially periodic array and a low-Re two-equation closure. Numerical Heat Transfer: Part A: Applications, 39(1):35-59.

Pokrajac, D. and Manes, C. (2008). Interface between turbulent flows above and within rough porous walls. Acta Geophysica, 56(3):824.

Prinos, P., Sofialidis, D., and Keramaris, E. (2003). Turbulent flow over and within a porous bed. Journal of Hydraulic Engineering, 129(9):720-733.

Rhie, C. and Chow, W. (1983). Numerical study of the turbulent flow past an airfoil with trailing edge separation. $A I A A$ journal, 21(11):1525-1532.

Silva, R. and de Lemos, M. (2003a). Numerical analysis of the stress jump interface condition for laminar flow over a porous layer. Numerical Heat Transfer: Part A: Applications, 43(6):603-617.

Silva, R. and de Lemos, M. (2003b). Turbulent flow in a channel occupied by a porous layer considering the stress jump at the interface. International Journal of Heat and Mass Transfer, 46(26):5113-5121.

Straatman, A., Gallego, N., Yu, Q., and Thompson, B. (2007). Characterization of porous carbon foam as a material for compact recuperators. Journal of Engineering for Gas Turbines and Power, 129(2):326-330.

Suga, K., Matsumura, Y., Ashitaka, Y., Tominaga, S., and Kaneda, M. (2010). Effects of wall permeability on turbulence. International Journal of Heat and Fluid Flow, 31(6):974-984.

Suga, K., Tominaga, S., Mori, M., and Kaneda, M. (2013). Turbulence characteristics in flows over solid and porous square ribs mounted on porous walls. Flow, turbulence and combustion, 91(1):19-40.

Vafai, K. and Tien, C. (1981). Boundary and inertia effects on flow and heat transfer in porous media. International Journal of Heat and Mass Transfer, 24(2):195-203.

Wang, L., Salewski, M., and Sundén, B. (2010). PIV measurements of turbulent flow in a channel with solid or perforated ribs. In ASME 2010 3rd Joint US-European Fluids Engineering Summer Meeting collocated with 8th International Conference on Nanochannels, Microchannels, and Minichannels, pages 1995-2003. American Society of Mechanical Engineers.

Whitaker, S. (1999). The method of volume averaging, volume 13. Springer Science \& Business Media. 